\title{
Zero-Hopf bifurcations in 3-dimensional differential systems with no equilibria
}

\author{
Murilo R. Cândido ${ }^{\mathrm{a}, *}$, Jaume Llibre ${ }^{\mathrm{a}}$ \\ ${ }^{a}$ Departament de Matemàtiques, Universitat Autònoma de Barcelona, 08193 Bellaterra, \\ Barcelona, Catalonia, Spain
}

\begin{abstract}
Recently sixteen 3-dimensional differential systems exhibiting chaotic motion and having no equilibria have been studied, and it has been graphically observed that these systems have a period-doubling cascade of periodic orbits providing a route to chaos. Here using new results on the averaging theory we prove that these systems exhibit, for some values of their parameters different to the ones having chaotic motion, either a zero-Hopf or a Hopf bifurcation, and graphically we observed that the periodic orbit starting in those bifurcations is at the beginning of the mentioned period-doubling cascade.
\end{abstract}

Keywords: Averaging theory, Periodic solutions, Quadratic polynomial differential system, Zero-Hopf bifurcation.

2010 MSC: 34C29, 37C27.

\section{Introduction and statement of the main results}

In general the equilibria of a chaotic nonlinear system play an important role in its dynamics. In fact, one of the most important methods for obtaining 3-dimensional chaotic systems is the Shilnikov's method [1], which using a homoclinic orbit from the intersection of the stable and unstable manifolds of a saddle-focus equilibrium point with specified eigenvalues, provides the existence of a horseshoe in the neighborhood of this orbit and, consequently the existence of chaotic motion.

However some particularly important natural phenomena are described by nonlinear systems having no equilibria. Such as, the Noose-Hover oscillator [2], the Wei system [3] and the Wang-Chen system [4]. These nonlinear systems present chaotic behavior that cannot be detected by Shilnikov's method.

The increasing interest in finding examples of simple chaotic flows without equilibria have been motivating many researchers in recent times, see for instance $[5,6,7,3,8]$. The theoretical and practical importance of these systems

\footnotetext{
* Corresponding author

Email addresses: candidomr@mat.uab.cat (Murilo R. Cândido), jllibre@mat.uab.cat (Jaume Llibre)
} 
converted this subject in a new attractive research direction. Although there is still little knowledge about the characteristics of such systems.

In this paper we shall study the existence of zero-Hopf bifurcations in 3dimensional systems, and graphically we will show that such bifurcations sometimes are the starting bifurcation of a rout to chaos. In general a zero-Hopf bifurcation is a codimension-two bifurcation of a 3-dimensional autonomous differential equation with a zero-Hopf equilibrium, and a zero-Hopf equilibrium of a 3-dimensional autonomous differential equation is an equilibrium point having two purely conjugate imaginary eigenvalues and a zero eigenvalue. Due to the lack of a general theory describing all the possible kinds of bifurcations that an unfolding of a zero-Hopf bifurcation can produce, most of the systems exhibiting these kind of bifurcations must be studied directly. In this paper we use averaging theory for detecting periodic solutions bifurcating from a zero-Hopf equilibrium. Furthermore, using Theorem 3 we were able to detect periodic solutions in very degenerate cases, for instance when the first averaged equation has a continuum of zeros.

In 2013 Jafari et al [5] have reported a catalogue of seventeen elementary three dimensional chaotic flows. This catalogue contains most of the elementary examples known of such systems and it includes the systems of the Noose-Hoover oscillator, the Wei system and the Wang-Chen system, listed there as system (1), (2) and (3), respectively. In [5] the authors used their own custom software to search for the algebraically simplest three-dimensional chaotic systems with quadratic nonlinearities and no equilibria. The search was inspired by the observation that each of the previously known examples of such systems contains a constant term (here represented by $a$ ), and that if the constant is set to zero, the resulting system is non-hyperbolic (the equilibria have eigenvalues with real part equal to zero). The method used to find these systems is that proposed in $[9]$.

In this paper we use recent results on averaging theory for studying the zeroHopf and the Hopf bifurcation of sixteen systems from the seventeen of [5], all of them have a parameter $a \in \mathbb{R}$. The averaging method could provide information about periodic solutions in all systems presented in [5] with the exception of their system SNE 14, because the present results on the averaging theory applied to such system do not provide any information about their periodic solutions. This is the reason that such differential system is not considered here.

$$
\begin{aligned}
& \dot{x}=y, \quad \dot{x}=-y, \quad \dot{x}=y, \\
& \dot{y}=-x-z y, \quad \text { (1) } \quad \dot{y}=x+z, \quad \text { (2) } \quad \dot{y}=z, \\
& \dot{z}=y^{2}-a . \quad \dot{z}=2 y^{2}+x z-a . \quad \dot{z}=0.1 x^{2}+1.1 x z-y+a \text {. } \\
& \dot{x}=-0.1 y+a, \quad \dot{x}=2 y, \quad \dot{x}=y, \\
& \dot{y}=x+z, \quad \text { (4) } \quad \dot{y}=2 x-z, \quad \text { (5) } \quad \dot{y}=z, \\
& \dot{z}=x z-3 y . \quad \dot{z}=-y^{2}+z^{2}+a . \quad \dot{z}=-y-x z-y z-a \text {. }
\end{aligned}
$$




$$
\begin{aligned}
& \dot{x}=y, \quad \dot{x}=y, \quad \dot{x}=y, \\
& \dot{y}=-x+z, \quad \text { (7) } \quad \dot{y}=-x-z y, \quad \text { (8) } \quad \dot{y}=-x-z y \text {, } \\
& \dot{z}=0.8 x^{2}+z^{2}+a . \quad \dot{z}=x y+0.5 x^{2}-a . \quad \dot{z}=-x z+7 x^{2}-a . \\
& \dot{x}=z, \\
& \dot{y}=z-y \text {, } \\
& \dot{z}=-0.9 y-x y+x z+a \text {. } \\
& \dot{x}=y, \\
& \dot{y}=-x+z \text {, } \\
& \dot{z}=-2 x y-1.8 x z+z-a \text {. } \\
& \dot{x}=z, \\
& \dot{y}=x-y, \\
& \dot{z}=-4 x^{2}+8 x y+y z+a \text {. } \\
& \dot{x}=y, \\
& \dot{y}=z \text {, } \\
& \dot{z}=x^{2}-y^{2}+x y+0.4 x z+a . \\
& \dot{z}=x y+x z+0.2 y z-a \text {. } \\
& \dot{x}=-0.8 x-0.5 y^{2}+x z+a, \\
& \dot{y}=-0.8 y-0.5 z^{2}+y x+a, \\
& \dot{z}=-0.8 z-0.5 x^{2}+z y+a \text {. } \\
& \dot{x}=-y-z^{2}+2.3 x y+a, \\
& \dot{y}=-z-x^{2}+2.3 y z+a \text {, } \\
& \dot{z}=-x-y^{2}+2.3 z x+a \text {. }
\end{aligned}
$$

Each of the systems (1)-(10) have an equilibrium that undergoes a zero-Hopf bifurcation at $a=a^{*}=0$, and no equilibria for $a>0$. Each of the systems (11)-(16) have an equilibrium that undergoes a Hopf bifurcation at some $a=a^{*}$. The limit cycle, which appears in this Hopf bifurcation, later on produces a period-doubling cascade, and finally a chaotic attractor with no equilibria, i.e. the equilibrium point which exhibits the Hopf bifurcation disappears before the chaotic attractor appears.

In this paper these sixteen chaotic flows are studied. Jafari et al [5] have reported numerically a period doubling cascade of periodic orbits originating the route to the chaotic motion in these systems. Here we graphically observe that the first periodic orbit performing the period doubling bifurcation detected by Jafari et al emerges in those systems at a zero-Hopf or Hopf bifurcation. This helps to understand the mechanism of chaos in these systems, and the objective of this paper is to show the existence of these zero-Hopf or Hopf bifurcations using the averaging theory.

One of the contributions of this work is to show that in many cases the periodic solutions that generate (via period-doubling) the chaotic attractor started with a periodic orbit coming from a zero-Hopf or a Hopf bifurcation. Using a suitable formulation of the averaging theory this paper complement the study of the zero-Hopf bifurcation started in [10].

The next theorem shows that the systems considered exhibit a zero-Hopf bifurcation at $a=0$. Although we can check that these systems have no equilibria 
when $a>0$.

Theorem 1. The following statements hold.

(i) The differential systems (1)-(10) exhibit a zero-Hopf bifurcation at $a=0$, more precisely for $a>0$ sufficiently small they have a periodic orbit which tends to a zero-Hopf equilibrium when $a \rightarrow 0$.

(ii) All the periodic solutions emerging in the zero-Hopf bifurcation are nonhyperbolic, with the exception of the differential system (2) that has a hyperbolic periodic solution.

(iii) All the periodic solutions in the zero-Hopf bifurcation emerge around the zero-Hopf equilibrium point located at the origin of coordinates, with the exception of system (10), which has the periodic solution emerging from the zero-Hopf equilibrium point $(1,0,0)$.

Another interesting aspect of some differential systems provided in [5] is that some of them have equilibria only if the parameter $a$ belongs to convenient intervals. In these intervals a Hopf bifurcation occurs and a periodic solution emerge in the system, but as $a$ increases the equilibria disappear and the isolated periodic solution coming from the Hopf bifurcation starts its cascade of perioddoubling. The differential systems having this behaviour are (11)-(16), in fact for $a<5 / 36$ system (11) has the equilibria

$$
P_{ \pm}=\left(\frac{1}{18}(5 \pm \sqrt{25-180 a}), 0, \frac{1}{18}(5 \pm \sqrt{25-180 a})\right),
$$

such that when $a=0$ the origin $\left(P_{-}\right)$is an equilibrium point with eigenvalues $\lambda_{1,2}= \pm i$ and $\lambda_{3}=-1$. Similarly, if $a<0$ system (12) has the equilibria

$$
P_{ \pm}=\left( \pm \frac{\sqrt{-a}}{2}, \pm \frac{\sqrt{-a}}{2}, 0\right)
$$

if $a=-196$ the equilibrium point $P_{+}$becomes a Hopf equilibrium with eigenvalues $\lambda_{1,2}= \pm i \sqrt{7}$ and $\lambda_{3}=-8$. System (13) has the equilibria

$$
P_{ \pm}=( \pm \sqrt{-a}, 0, \pm \sqrt{-a})
$$

for $a<0$. When $a=-25 / 16$ the equilibria $P_{+}$is a Hopf equilibrium with eigenvalues $\lambda_{1,2}= \pm i \sqrt{2}$ and $\lambda_{3}=-5 / 4$. System (14) has the equilibria

$$
P_{ \pm}=( \pm \sqrt{-a}, 0,0)
$$

for $a<0$ and for $a=-25$ the equilibria $P_{+}$becomes a Hopf equilibrium with eigenvalues $\lambda_{1,2}= \pm i \sqrt{5}$ and $\lambda_{3}=-2$. System (15) is considered after the statement of Theorem 2. Finally, system (16) has the equilibria

$$
P_{ \pm}=\left(\frac{1}{13}(5 \pm \sqrt{25-130 a}), 0, \frac{1}{13}(5 \pm \sqrt{25-130 a})\right),
$$

for $a<5 / 26$ and when $a=-560 / 1849$ the equilibrium point $P_{-}$is Hopf equilibrium with eigenvalues $\lambda_{1,2}= \pm i \sqrt{3}$ and $\lambda_{3}=-69 / 43$. 
Theorem 2. Consider the differential systems (11)-(16). The following statements hold for $\varepsilon \neq 0$ sufficiently small with $a_{2}>0$.

(i) Let $a=a_{2} \varepsilon^{2}$. System (11) has a Hopf bifurcation at $a=0$ and a periodic solution emerges from the origin of coordinates of this system.

(ii) Let $a=-196+a_{2} \varepsilon^{2}$. System (12) has a Hopf bifurcation at $a=-196$ and a periodic solution emerges from the equilibrium point $(-7,-7,0)$.

(iii) Let $a=-\frac{25}{16}+a_{2} \varepsilon^{2}$. System (13) has a Hopf bifurcation at $a=-\frac{25}{16}$ and a periodic solution emerges from the equilibrium point $\left(-\frac{5}{4}, 0, \frac{5}{4}\right)$.

(iv) Let $a=-25+a_{2} \varepsilon^{2}$. System (14) has a Hopf bifurcation at $a=-25$ and a periodic solution emerges from the equilibrium point $(-5,0,0)$.

(v) Let $a=\frac{8}{25}+a_{2} \varepsilon^{2}$. System (15) has a zero-Hopf bifurcation at $a=\frac{8}{25}$ and a periodic solution emerges from the equilibrium point $\left(\frac{4}{5}, \frac{4}{5}, \frac{4}{5}\right)$.

(vi) Let $a=-\frac{560}{1849}+a_{2} \varepsilon^{2}$. System (16) has a Hopf bifurcation at $a=-\frac{560}{1849}$ and a periodic solution emerges from the equilibrium point $\left(-\frac{10}{43},-\frac{10}{43},-\frac{10}{43}\right)$.

To illustrate graphically the relation between the periodic solutions provided by Theorem 2 and the chaotic attractors presented in [5] we shall use system (16) as an example. First we observe that for $a<5 / 26$ the system has the following equilibrium point

$$
p_{0}=\left(\frac{1}{13}(5-\sqrt{25-130 a}), \frac{1}{13}(5-\sqrt{25-130 a}), \frac{1}{13}(5-\sqrt{25-130 a})\right) .
$$

Taking $a=-\frac{560}{1849}+a_{2} \varepsilon^{2}$ and $\varepsilon>0$ sufficiently small system (16) has a periodic solution as stated by Theorem $2(v i)$. In this case the equilibirum point $p_{0}$ exists only if $0<\varepsilon<\frac{23}{2} \sqrt{\frac{15}{6799}} \approx 0.54$. For instance, taking $a_{2}=-\frac{560}{1849}+2$ and $\varepsilon=0.002$ it can be seen that the solution of system (16) starting at $(1,-1,0)$ converges to the periodic solution, see Figure 1 . Increasing the value of $\varepsilon$, for instance $\varepsilon=0.251$ and $\varepsilon=0.511$, the periodic solution increases its size and still remains stable, see Figures 2 and 3 respectively. For all the previously values of $\varepsilon$ the point $p_{0}$ is an equibilibrium point of system (16). However, for $\varepsilon=0.691$ and $\varepsilon=0.97$ the system has no equilibria and we can see that the periodic solution starts its cascade of period-doubling, see Figure 4 and 5. Taking $\varepsilon=1$ the system has a strange attractor as it is reported in [5], see Figure 6. These solutions were plotted for $0 \leq t \leq 1000$. 

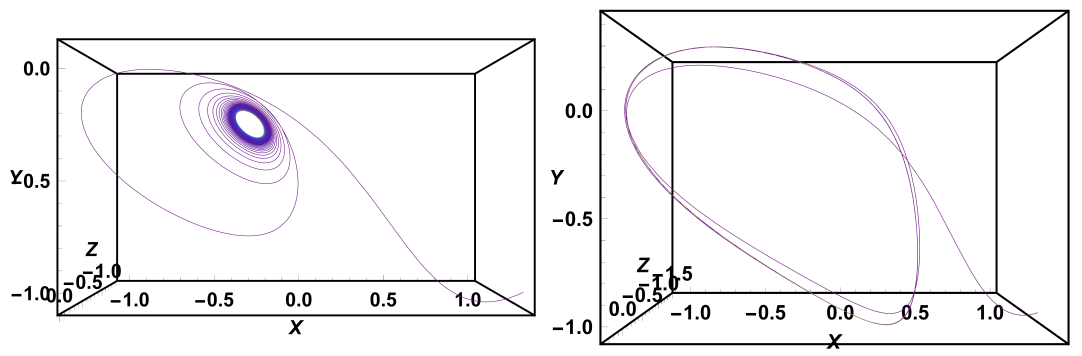

Figure 1

Figure 2
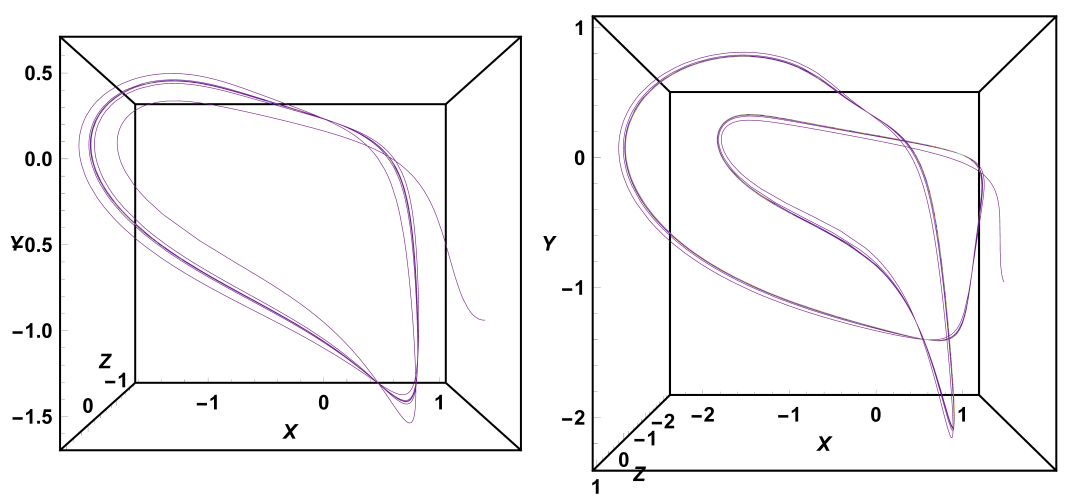

Figure 3

Figure 4 


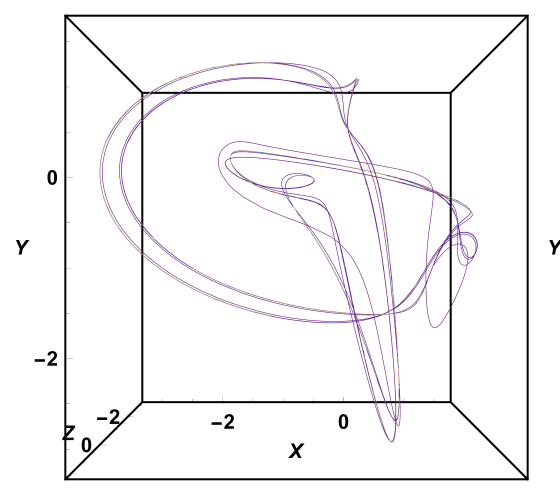

Figure 5

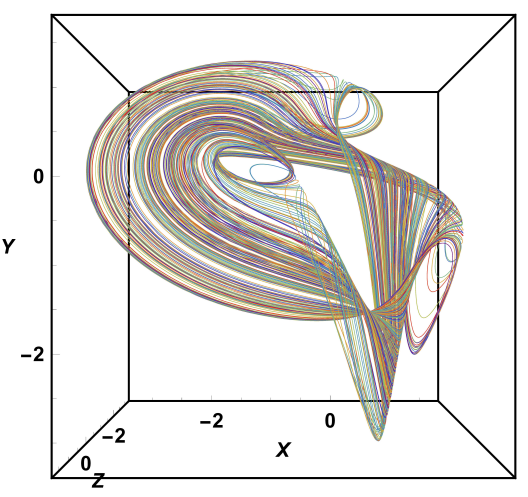

Figure 6

In the next section we present the proofs of our results.

\section{The averaging theory}

The averaging theory is one of the classical tools for studying the solutions of the nonlinear dynamical systems, and in particular their periodic solutions [11]. Here it will be used for proving Theorems 1 and 2 .

We consider the differential systems in the normal form for applying averaging theory

$$
\dot{\mathbf{x}}=\mathbf{F}_{0}(t, \mathbf{x})+\varepsilon \mathbf{F}_{1}(t, \mathbf{x})+\varepsilon^{2} \mathbf{F}_{2}(t, \mathbf{x})+\varepsilon^{3} \mathbf{F}_{3}(t, \mathbf{x})+\varepsilon^{4} \widetilde{\mathbf{F}}(t, \mathbf{x}, \varepsilon),
$$

with $\mathbf{x}$ in some open $\Omega$ subset of $\mathbb{R}^{n}, t \in[0, \infty), \varepsilon \in\left[-\varepsilon_{0}, \varepsilon_{0}\right]$. We assume $\mathbf{F}_{i}$ and $\widetilde{\mathbf{F}}$ for all $i=1,2,3$ are $T$-periodic in the variable $t$. Let $\mathbf{x}(t, z, 0)$ be the solution of the unperturbed system

$$
\dot{\mathbf{x}}=\mathbf{F}_{0}(t, \mathbf{x})
$$

such that $\mathbf{x}(0, \mathbf{z}, 0)=\mathbf{z}$. We define $M(t, \mathbf{z})$ the fundamental matrix of the linear differential system

$$
\dot{\mathbf{y}}=\frac{\partial \mathbf{F}_{0}(t, \mathbf{x}(t, \mathbf{z}, 0))}{\partial \mathbf{x}} \mathbf{y} .
$$

The displacement map of system (17) is defined as

$$
\mathbf{d}(\mathbf{z}, \varepsilon)=\mathbf{x}(T, \mathbf{z}, \varepsilon)-\mathbf{z}
$$

In order to have $\mathbf{d}(\mathbf{z}, \varepsilon)$ well defined we assume that

(H) For $|\varepsilon| \neq 0$ sufficiently small, there exists an open set $U \subset \Omega$ such that for all $\mathbf{z} \in U$ the solution $\mathbf{x}(t, \mathbf{z}, \varepsilon)$ is defined on the interval $\left[0, t_{(\mathbf{z}, \varepsilon)}\right)$ with $t_{(\mathbf{z}, \varepsilon)}>T$. 
This hypothesis is always true when the unperturbed system has a manifold of $T$-periodic solutions in $U$. Writing the displacement map (18) in power series of $\varepsilon$ we obtain

$$
\mathbf{d}(\mathbf{z}, \varepsilon)=\mathbf{g}_{0}(\mathbf{z})+\varepsilon \mathbf{g}_{1}(\mathbf{z})+\varepsilon^{2} \mathbf{g}_{2}(\mathbf{z})+\varepsilon^{3} \mathbf{g}_{3}(\mathbf{z})+\varepsilon^{4} \widetilde{\mathbf{g}}(\mathbf{z}, \varepsilon),
$$

where for $i=0,1,2,3$ we have

$$
\mathbf{g}_{i}(\mathbf{z})=M(T, \mathbf{z})^{-1} \frac{\mathbf{y}_{i}(T, \mathbf{z})}{i !}
$$

being

$$
\begin{aligned}
\mathbf{y}_{0}(t, z)= & \mathbf{x}(t, \mathbf{z}, 0)-\mathbf{z}, \\
\mathbf{y}_{1}(t, \mathbf{z})= & M(t, \mathbf{z}) \int_{0}^{t} M(\tau, \mathbf{z})^{-1} \mathbf{F}_{1}(\tau, \mathbf{x}(\tau, \mathbf{z}, 0)) \mathrm{d} \tau \\
\mathbf{y}_{2}(t, \mathbf{z})= & M(t, \mathbf{z}) \int_{0}^{t} M(\tau, \mathbf{z})^{-1}\left[2 \mathbf{F}_{2}(\tau, \mathbf{x}(\tau, \mathbf{z}, 0))+2 \frac{\partial \mathbf{F}_{1}}{\partial \mathbf{x}}(\tau, \mathbf{x}(\tau, \mathbf{x}, 0)) \mathbf{y}_{1}(\tau, \mathbf{z})\right. \\
& \left.+\frac{\partial^{2} \mathbf{F}_{0}}{\partial \mathbf{x}^{2}}(\tau, \mathbf{x}(\tau, \mathbf{z}, 0)) \mathbf{y}_{1}(\tau, \mathbf{z})^{2}\right] \mathrm{d} \tau, \\
\mathbf{y}_{3}(t, \mathbf{z})= & M(t, \mathbf{z}) \int_{0}^{t} M(\tau, \mathbf{z})^{-1}\left[6 \mathbf{F}_{3}(\tau, \mathbf{x}(\tau, \mathbf{z}, 0))+6 \frac{\partial \mathbf{F}_{2}}{\partial \mathbf{x}}(\tau, \mathbf{x}(\tau, \mathbf{z}, 0)) \mathbf{y}_{1}(\tau, \mathbf{z})\right. \\
& +3 \frac{\partial^{2} \mathbf{F}_{1}}{\partial \mathbf{x}^{2}}(\tau, \mathbf{x}(\tau, \mathbf{z}, 0)) \mathbf{y}_{1}(\tau, \mathbf{z})^{2}+3 \frac{\partial \mathbf{F}_{1}}{\partial \mathbf{x}}(\tau, \mathbf{x}(\tau, \mathbf{z}, 0)) \mathbf{y}_{2}(\tau, \mathbf{z}) \\
& +3 \mathbf{y}_{2}(\tau, \mathbf{z})^{T} \frac{\partial^{2} \mathbf{F}_{0}}{\partial \mathbf{x}^{2}}(\tau, \mathbf{x}(\tau, \mathbf{z}, 0)) \mathbf{y}_{1}(\tau, \mathbf{z}) \\
& \left.+\frac{\partial^{3} \mathbf{F}_{0}}{\partial \mathbf{x}^{3}}(\tau, \mathbf{x}(\tau, \mathbf{z}, 0)) \mathbf{y}_{1}(\tau, \mathbf{z})^{3}\right] \mathrm{d} \tau .
\end{aligned}
$$

The functions $\mathbf{g}_{1}, \mathbf{g}_{2}$ and $\mathbf{g}_{3}$ will be called here the averaged functions of system (17).

We say that system (17) has a periodic solution bifurcating from the point $\mathbf{z}_{0}$ if there exists a branch of solutions $\mathbf{z}(\varepsilon)$ for the displacement function such that $\mathbf{d}(\mathbf{z}(\varepsilon), \varepsilon)=\mathbf{0}$ and $\mathbf{z}(0)=\mathbf{z}_{0}$.

Now we will state the theorem about the existence of periodic solutions of system (17). The methodology used here was introduced for studying systems such that the unperturbed part has a sub-manifold of $T$-periodic solutions, see for instance [12] and [13]. Here we write the result in a way that make it more adaptable and easy to apply. This result has been used for studying bifurcation of periodic orbits into the Lorenz and FitzHugh-Nagumo system [14]. In [15] this formulation is presented in a more general way. Assume $m<n$, and let $\pi: \mathbb{R}^{m} \times \mathbb{R}^{n-m} \rightarrow \mathbb{R}^{m}$ and $\pi^{\perp}: \mathbb{R}^{m} \times \mathbb{R}^{n-m} \rightarrow \mathbb{R}^{n-m}$ denote the projections onto the first $m$ coordinates and onto the last $n-m$ coordinates, respectively. 
For a point $\mathbf{z} \in U$ we also consider $\mathbf{z}=(a, b) \in \mathbb{R}^{m} \times \mathbb{R}^{n-m}$. Consider the graph

$$
\mathcal{Z}=\left\{\mathbf{z}_{\alpha}=(\alpha, \beta(\alpha)): \alpha \in \bar{V}\right\} \subset U,
$$

where $V$ is an open set of $\mathbb{R}^{m}$ and $\beta: \bar{V} \rightarrow \mathbb{R}^{n-m}$ is a $\mathcal{C}^{4}$ function.

Theorem 3. Let $r \in\{0,1\}$ such that $\mathbf{g}_{r}$ is the first nonzero averaged function. In addition to hypothesis $(H)$ assume that

(i) The averaged function $\mathbf{g}_{r}$ vanishes on the graph (20). That is $\mathbf{g}_{r}\left(\mathbf{z}_{\alpha}\right)=\mathbf{0}$ for all $\alpha \in \bar{V}$.

(ii) The Jacobian matrix

$$
D \mathbf{g}_{r}\left(\mathbf{z}_{\alpha}\right)=\left(\begin{array}{cc}
\Lambda_{\alpha} & \Gamma_{\alpha} \\
B_{\alpha} & \Delta_{\alpha}
\end{array}\right)
$$

where $\Lambda_{\alpha}=D_{a} \pi g_{r}\left(z_{\alpha}\right), \Gamma_{\alpha}=D_{b} \pi g_{r}\left(z_{\alpha}\right), B_{\alpha}=D_{a} \pi^{\perp} g_{r}\left(z_{\alpha}\right)$ and $\Delta_{\alpha}=$ $D_{b} \pi^{\perp} g_{r}\left(z_{\alpha}\right)$. Satisfies $\operatorname{det}\left(\Delta_{\alpha}\right) \neq 0$ for all $\alpha \in \bar{V}$.

We define the functions

$$
\begin{aligned}
& f_{1}(\alpha)=-\Gamma_{\alpha} \Delta_{\alpha}^{-1} \pi^{\perp} \mathbf{g}_{r+1}\left(\mathbf{z}_{\alpha}\right)+\pi \mathbf{g}_{r+1}\left(\mathbf{z}_{\alpha}\right) \\
& f_{2}(\alpha)=\frac{1}{2} \Gamma_{\alpha} \gamma_{2}(\alpha)+\frac{1}{2} \frac{\partial^{2} \pi \mathbf{g}_{r}}{\partial b^{2}}\left(\mathbf{z}_{\alpha}\right) \gamma_{2}(\alpha)^{2}+\frac{\partial \pi \mathbf{g}_{r+1}}{\partial b}\left(z_{\alpha}\right) \gamma_{2}(\alpha)+\pi \mathbf{g}_{r+2}\left(\mathbf{z}_{\alpha}\right) \\
& \gamma_{2}(\alpha)=-\Delta_{\alpha}^{-1} \pi^{\perp} \mathbf{g}_{r+1}\left(\mathbf{z}_{\alpha}\right) \\
& \gamma_{2}(\alpha)=-\Delta_{\alpha}^{-1}\left(\frac{\partial^{2} \pi^{\perp} \mathbf{g}_{r}}{\partial b^{2}}\left(\mathbf{z}_{\alpha}\right) \gamma_{2}(\alpha)^{2}+2 \frac{\partial \pi^{\perp} \mathbf{g}_{r+1}}{\partial b}\left(\mathbf{z}_{\alpha}\right) \gamma_{2}(\alpha)+2 \pi^{\perp} \mathbf{g}_{r+2}(\alpha)\right) .
\end{aligned}
$$

Then the following statements hold.

(a) If there exists $\alpha^{*} \in V$ such that $f_{1}\left(\alpha^{*}\right)=0$ and $\operatorname{det}\left(D f_{1}\left(\alpha^{*}\right)\right) \neq 0$ then for $|\varepsilon| \neq 0$ sufficiently small, there is an initial condition $\mathbf{z}(\varepsilon) \in U$ such that $\mathbf{z}(0)=\mathbf{z}_{\alpha^{*}}$ and the solution $\mathbf{x}(t, \mathbf{z}(\varepsilon), \varepsilon)$ of system (17) is T-periodic.

(b) Assume that $f_{1} \equiv 0$. If there exists $\alpha^{*} \in V$ such that $f_{2}\left(\alpha^{*}\right)=0$ and $\operatorname{det}\left(D f_{2}\left(\alpha^{*}\right)\right) \neq 0$, for $|\varepsilon| \neq 0$ sufficiently small there is an initial condition $\mathbf{z}(\varepsilon) \in U$ such that $\mathbf{z}(0)=\mathbf{z}_{\alpha^{*}}$ and the solution $\mathbf{x}(t, \mathbf{z}(\varepsilon), \varepsilon)$ of system (17) is T-periodic.

Consider $r \in\{0,1\}, \alpha=\mathbf{z}$ and the hypothesis $(H)$. Thus the result of the above theorem follows even assuming that $\mathbf{g}_{r} \equiv 0$. In this case we take $\pi$ and $\pi^{\perp}$ as the identity and the null operator respectively and no assumptions about $\Delta_{\alpha}$. Then we have the following corollary.

Corollary 4. For $r \in\{0,1\}$ assume that $\mathbf{g}_{r} \equiv 0$. If there exists $\mathbf{z}^{*} \in \Omega$ such that $\mathbf{g}_{r+1}\left(\mathbf{z}^{*}\right)=0$ and $D \mathbf{g}_{r+1}\left(\mathbf{z}^{*}\right) \neq 0$ then there exists a T-periodic solution $\mathbf{x}(t, \mathbf{z}(\varepsilon), \varepsilon)$ for system (17) such that $\mathbf{z}(0)=\mathbf{z}^{*}$. 
The averaging theory allows to find periodic solutions for periodic nonautonomous differential systems. However here we are interested in using it for studying the periodic solutions bifurcating from a zero-Hopf equilibrium point of the autonomous differential systems (1)-(16). The algorithm for doing that is the following.

(i) First we must identify the conditions for which these systems have a zeroHopf equilibrium. In this paper the zero-Hopf equilibrium usually happens when $a=0$.

(ii) We translate the zero-Hopf equilibrium point at the origin the origin of coordinates and scale the system with a small parameter $\varepsilon$ when it is necessary, because the zero-Hopf and the Hopf bifurcation and the averaging theory needs a such small parameter.

(iii) We write the system in the cylindrical coordinates $(\rho, \theta, z)$ where $(x, y, z)=$ $(\rho \cos \theta, \rho \sin \theta, z)$.

(iv) We take the angular variable $\theta$ as the new independent variable of the differential system. Obtaining a 2-dimensional periodic non-autonomous system $\frac{d \rho}{d \theta}=\ldots, \frac{d z}{d \theta}=\ldots$ in the variable $\theta$. In this way the differential system is written into the normal form for applying to it the averaging theory for obtaining the periodic solutions.

(v) Going back through the change of variables we get the periodic solutions bifurcating from the zero-Hopf equilibrium.

\section{Proof of Theorem 1}

The proof of Theorem 1 for systems (1)-(4) and (6)-(10) can be obtained using Corollary 4 with $r=0$ which is equivalent with the classical averaging theory as we shall see. We start proving Theorem 1 for system (10).

Proof of Theorem 1 for system (10). We take $a=a_{2} \varepsilon^{2}$ with $a_{2}>0$ and $\varepsilon>0$ sufficiently small. First we translate the point $p=(1,0,0)$ to the origin of coordinates then we use the change of variables

$$
(x, y, z)=\varepsilon\left(\frac{19 X}{9}+Z, X-\frac{\sqrt{10} Y}{3}, \frac{19 \sqrt{10} Y}{30}\right),
$$

into the differential system (10) writes

$$
\begin{aligned}
& \dot{X}=-\frac{3 Y}{\sqrt{10}}+\frac{1}{171} \varepsilon\left((10 X+3 \sqrt{10} Y)(19 X+9 Z)-90 a_{2}\right), \\
& \dot{Y}=\frac{3 X}{\sqrt{10}}+\frac{\varepsilon\left((10 X+3 \sqrt{10} Y)(19 X+9 Z)-90 a_{2}\right)}{57 \sqrt{10}}
\end{aligned}
$$




$$
\dot{Z}=\frac{1}{81} \varepsilon\left(90 a_{2}-(10 X+3 \sqrt{10} Y)(19 X+9 Z)\right) .
$$

Using the cylindrical change of variables $(X, Y, Z)=(\rho \cos \theta, \rho \sin \theta, z)$ where $\rho>0$, system (21) writes

$$
\begin{aligned}
\dot{\rho}= & \frac{1}{342} \varepsilon\left(-18 a_{2}(3 \sqrt{10} \sin \theta+10 \cos \theta)+9 \rho z(6 \sqrt{10} \sin (2 \theta)+\cos (2 \theta)+19)\right. \\
& \left.+19 \rho^{2} \cos \theta(6 \sqrt{10} \sin (2 \theta)+\cos (2 \theta)+19)\right), \\
\dot{\theta}= & \frac{3}{\sqrt{10}}+\frac{\varepsilon}{1710 \rho}\left(\rho(3 \sqrt{10} \sin \theta+10 \cos \theta)(19 \rho \cos \theta+9 z)-90 a_{2}\right) \\
& (3 \sqrt{10} \cos \theta-10 \sin \theta), \\
\dot{z}= & \frac{1}{81} \varepsilon\left(90 a_{2}-\rho(3 \sqrt{10} \sin \theta+10 \cos \theta)(19 \rho \cos \theta+9 z)\right) .
\end{aligned}
$$

This differential system can be reduced to the normal form for applying the averaging theory. Taking $\theta$ as the new independent variable we obtain the differential system

$$
\begin{aligned}
\rho^{\prime}= & \frac{\varepsilon}{513} \sqrt{\frac{5}{2}}(\rho(6 \sqrt{10} \sin (2 \theta)+\cos (2 \theta)+19)(19 \rho \cos \theta+9 z) \\
& \left.-18 a_{2}(3 \sqrt{10} \sin \theta+10 \cos \theta)\right)+\mathcal{O}\left(\varepsilon^{2}\right), \\
z^{\prime}= & \frac{\varepsilon}{243} \sqrt{10}\left(90 a_{2}-r(3 \sqrt{10} \sin \theta+10 \cos \theta)(19 r \cos \theta+9 z)\right)+\mathcal{O}\left(\varepsilon^{2}\right),
\end{aligned}
$$

here the derivatives are taken with respect to $\theta$. Using (19) we write the functions $\mathbf{g}_{0} \equiv 0$ and $\mathbf{g}_{1}(\mathbf{z})=\sqrt{10} \pi\left(\frac{1}{3} \rho z, \frac{10}{243}\left(18 a_{2}-19 \rho^{2}\right)\right)$. The averaged function $\mathbf{g}_{1}$ has the solutions $\mathbf{z}_{ \pm}= \pm\left(3 \sqrt{\frac{2 a_{2}}{19}}, 0\right)$. The result follows by taking $r=0$ and $\mathbf{z}^{*}=\mathbf{z}_{+}$and applying Corollary 4 . The periodic solution is nonhyperbolic. The eigenvalues of the Jacobian matrix $D \mathbf{g}_{1}\left(\mathbf{z}_{+}\right)$are $\pm \frac{20}{9} i \sqrt{a_{2}}$.

Proof of Theorem 1 for systems (1)-(4) and (6)-(9). The proof of Theorem 1 for systems (1)-(4) and (6)-(9) is similar to the proof of Theorem 1 for system (10). It can be done using Corollary 4 with $r=0$ and analogous computations. The reader can check in Theorem 1.1 of [10] the proofs for these systems using classical first order averaging. The authors also provide approximations for the periodic solutions found.

Now we prove Theorem 1 for system (5). This proof is not provided in [10] because the classical averaging theory does not provide information for this case. We shall prove this result using statement $(b)$ of Theorem 3. 
Proof of Theorem 1 for system (5). Using the change of variables $(x, y, z)=$ $\varepsilon(x+y,-y,-2 z)$ the differential system (5) writes

$$
\begin{aligned}
& \dot{X}=-2 Y+\frac{1}{2} \varepsilon\left(a_{2}-Y^{2}+4 Z^{2}\right), \\
& \dot{Y}=2 X, \\
& \dot{Z}=\frac{1}{2} \varepsilon\left(-a_{2}+Y^{2}-4 Z^{2}\right) .
\end{aligned}
$$

Using the cylindrical change of variables $(X, Y, Z)=(\rho \cos \theta, \rho \sin \theta, z)$ where $\rho>0$, system (22) becomes

$$
\begin{aligned}
& \dot{\rho}=\varepsilon \frac{1}{2} \cos \theta\left(a_{2}-\rho^{2} \sin ^{2} \theta+4 z^{2}\right), \\
& \dot{\theta}=2-\varepsilon \frac{\sin \theta\left(a_{2}-\rho^{2} \sin ^{2} \theta+4 z^{2}\right)}{2 \rho}, \\
& \dot{z}=\varepsilon \frac{1}{2}\left(-a_{2}+\rho^{2} \sin ^{2} \theta-4 z^{2}\right) .
\end{aligned}
$$

This differential system can be reduced to the normal form for applying averaging theory. Taking $\theta$ as the new independent variable we obtain the differential system

$$
\begin{aligned}
\rho^{\prime}= & \varepsilon \frac{1}{4} \cos \theta\left(a_{2}-\rho^{2} \sin ^{2} \theta+4 z^{2}\right)+\varepsilon^{2} \frac{\sin \theta \cos \theta\left(a_{2}-\rho^{2} \sin ^{2} \theta+4 z^{2}\right)^{2}}{16 \rho} \\
& +\varepsilon^{3} \frac{\sin ^{2} \theta \cos \theta\left(a_{2}-\rho^{2} \sin ^{2} \theta+4 z^{2}\right)^{3}}{64 \rho^{2}}+\mathcal{O}\left(\varepsilon^{4}\right), \\
z^{\prime}= & \varepsilon \frac{1}{4}\left(-a_{2}+\rho^{2} \sin ^{2} \theta-4 z^{2}\right)+\varepsilon^{2}-\frac{\sin \theta\left(a_{2}-\rho^{2} \sin ^{2} \theta+4 z^{2}\right)^{2}}{16 \rho} \\
& +\varepsilon^{3} \frac{\sin ^{2} \theta\left(-a_{2}+\rho^{2} \sin ^{2} \theta-4 z^{2}\right)^{3}}{64 \rho^{2}}+\mathcal{O}\left(\varepsilon^{4}\right),
\end{aligned}
$$

here the derivatives are taken with respect to $\theta$. Using (19) we write the functions

$$
\begin{aligned}
\mathbf{g}_{0}(\mathbf{z})= & (0,0) \\
\mathbf{g}_{1}(\mathbf{z})= & \pi\left(0,-\frac{a_{2}}{2}+\frac{\rho^{2}}{4}-2 z^{2}\right) \\
\mathbf{g}_{2}(\mathbf{z})= & \left(0, \frac{1}{2} \pi^{2} z\left(2 a_{2}-\rho^{2}+8 z^{2}\right)\right) \\
\mathbf{g}_{3}(\mathbf{z})= & \left(\frac { \pi } { 9 6 \rho } \left(24 a_{2}^{2}(\rho+z)+4 a_{2}\left(-7 \rho^{3}+48 z^{3}+96 \rho z^{2}-12 \rho^{2} z\right)+8 \rho^{5}+384 z^{5}\right.\right. \\
& +1152 \rho z^{4}-192 \rho^{2} z^{3}-208 \rho^{3} z^{2}+15 \rho^{4} z, \frac{\pi}{4608 \rho}\left(3 \rho \left(8\left(15-32 \pi^{2}\right) a_{2}^{2}\right.\right.
\end{aligned}
$$




$$
\begin{aligned}
& \left.+4\left(64 \pi^{2}-23\right) a_{2} \rho^{2}+\left(5-64 \pi^{2}\right) \rho^{4}\right)-128 z\left(9 a_{2}^{2}-18 a_{2} \rho^{2}+10 \rho^{4}\right) \\
& +9216 z^{3}\left(\rho^{2}-a_{2}\right)+48 \rho z^{2}\left(\left(60-256 \pi^{2}\right) a_{2}+\left(128 \pi^{2}-47\right) \rho^{2}\right) \\
& \left.\left.-18432 z^{5}+1152\left(5-32 \pi^{2}\right) \rho z^{4}\right)\right)
\end{aligned}
$$

Consider the graph $\mathcal{Z}=\left\{\mathbf{z}_{\alpha}=(\alpha, \beta(\alpha)): \beta(\alpha)=\sqrt{\frac{\alpha^{2}-2 a_{2}}{8}}\right.$ and $\left.\alpha \geq \sqrt{2 a_{2}}\right\}$. For all $\alpha \geq \sqrt{2 a_{2}}$ the averaged function $\mathbf{g}_{1}\left(\mathbf{z}_{\alpha}\right)=(0,0)$. Then taking $r=1$ in Theorem 3 we compute the bifurcation functions $f_{1}(\alpha)=0$ and $f_{2}(\alpha)=$ $\frac{\pi \alpha^{2}}{64} \sqrt{\frac{\alpha^{2}-2 a_{2}}{2}}$. For $\alpha^{*}=\sqrt{2 a_{2}}$ we have $f_{2}\left(\alpha^{*}\right)=0$ and the derivative of $f_{2}$ goes to infinity at $\alpha^{*}$, so it is a simple zero of $f_{2}$. Thus applying statement $(b)$ of Theorem 3 we have that system (23) has a periodic solution bifurcating from point $\mathbf{z}_{\alpha}^{*}$. Consequently, going back through the change of variables we have the existence of a periodic solution of system (5).

\section{Proof of Theorem 2}

Proof of Theorem 2 statement $(i)$. Using the change of variables $(x, y, z)=\varepsilon(X+$ $Z,-Y+Z, 2 Z)$ the differential system (11) writes

$$
\begin{aligned}
\dot{X} & =-Y+\frac{\varepsilon}{10}\left(5 a_{2}-2(X+Z)(5 Y-14 Z)\right), \\
\dot{Y} & =X+\frac{\varepsilon}{10}\left(2(X+Z)(5 Y-14 Z)-5 a_{2}\right), \\
\dot{Z} & =Z+\frac{\varepsilon}{10}\left(2(X+Z)(5 Y-14 Z)-5 a_{2}\right) .
\end{aligned}
$$

Using the cylindrical change of variables $(X, Y, Z)=(\rho \cos \theta, \rho \sin \theta, z)$ where $\rho>0$, system (24) becomes

$$
\begin{aligned}
& \dot{\rho}=\frac{\varepsilon}{10} \varepsilon(\cos \theta-\sin \theta)\left(5 a_{2}+28 \rho z \cos \theta-10 \rho \sin \theta(\rho \cos \theta+z)+28 z^{2}\right), \\
& \dot{\theta}=1+\frac{\varepsilon}{10 \rho}(\sin \theta+\cos \theta)\left(-5 a_{2}-28 \rho z \cos \theta+10 \rho \sin \theta(\rho \cos \theta+z)-28 z^{2}\right), \\
& \dot{z}=z+\frac{\varepsilon}{10}\left(2(5 \rho \sin \theta-14 z)(\rho \cos \theta+z)-5 a_{2}\right) .
\end{aligned}
$$

This differential system can be reduced to the normal form for applying the averaging theory. Taking $\theta$ as the new independent variable we obtain the differential system

$$
\begin{aligned}
\rho^{\prime}= & \frac{\varepsilon}{10}(\cos \theta-\sin \theta)\left(5 a_{2}+28 z^{2}+28 \rho z \cos \theta-10 \rho \sin \theta(\rho \cos \theta+z)\right) \\
& +\varepsilon^{2} \frac{(\cos \theta-\sin \theta)(\sin \theta+\cos \theta)}{100 \rho}\left(5 a_{2}-5 \rho^{2} \sin (2 \theta)+28 z^{2}-10 \rho z \sin \theta\right. \\
& +28 \rho z \cos \theta)^{2}+\mathcal{O}\left(\varepsilon^{3}\right),
\end{aligned}
$$




$$
\begin{aligned}
z^{\prime}= & z+\varepsilon\left(-5 a_{2}-28 z^{2}-28 \rho z \cos \theta+10 \rho \sin \theta(\rho \cos \theta+z)\right) \\
& \frac{(\rho-z(\sin \theta+\cos \theta))}{10 \rho}+\varepsilon^{2} \frac{(\sin \theta+\cos \theta)(-\rho+z \sin \theta+z \cos \theta)}{100 \rho^{2}} \\
& \left(5 a_{2}-5 \rho^{2} \sin (2 \theta)+28 z^{2}-10 \rho z \sin \theta+28 \rho z \cos \theta\right)^{2}+\mathcal{O}\left(\varepsilon^{3}\right),
\end{aligned}
$$

here the derivatives are taken with respect to $\theta$. Using (19) we write the functions

$$
\begin{aligned}
\mathbf{g}_{0}(\mathbf{z})= & \left(0,\left(1-e^{-2 \pi}\right) z\right) \\
\mathbf{g}_{1}(\mathbf{z})= & \left(\frac{1}{25}\left(e^{2 \pi}-1\right) z\left(71 \rho+42\left(1+e^{2 \pi}\right) z\right)\right. \\
& \left.\frac{\left(e^{2 \pi}-1\right)}{50 \rho}\left(-25 a_{2} \rho+10 \rho^{3}+28\left(e^{2 \pi}+e^{4 \pi}\right) z^{3}-94 e^{2 \pi} \rho z^{2}\right)\right) \\
\mathbf{g}_{2}(\mathbf{z})= & \left(-\frac{a_{2}}{7800 \rho}\left(156\left(-71+71 e^{2 \pi}-95 \pi\right) \rho^{2}+28\left(127-195 e^{4 \pi}+68 e^{6 \pi}\right) z^{2}\right.\right. \\
& \left.+3\left(1591-6474 e^{2 \pi}+4883 e^{4 \pi}\right) \rho z\right)+\frac{1}{26002860000 \rho}(52005720 \\
& \left(-284+284 e^{2 \pi}+5 \pi\right) \rho^{4}+\left(e^{8 \pi}\left(4823-2226 e^{2 \pi}+806 e^{6 \pi}\right)-3403\right) z^{4} \\
& 8453760+38584\left(e^{6 \pi}\left(1308320+1767897 e^{2 \pi}-1169940 e^{4 \pi}+90712 e^{6 \pi}\right)\right. \\
& -1996989) \rho z^{3}-\left(759163+666540 e^{4 \pi}-1872448 e^{6 \pi}+446745 e^{8 \pi}\right) \\
& \left.100011 \rho^{2} z^{2}+8000880\left(e^{\pi}-1\right)\left(1+e^{\pi}\right)\left(4799+10883 e^{2 \pi}\right) \rho^{3} z\right), \\
& -\frac{e^{3 \pi} a_{2}^{2} z \sinh (\pi)}{10 \rho^{2}}+\frac{1}{38646750675000 \rho^{2}}\left(6956415121500\left(e^{2 \pi}-1\right) \rho^{5}\right. \\
& +61590200 e^{2 \pi}\left(e^{8 \pi}\left(1258803-454104 e^{2 \pi}-409955 e^{4 \pi}+230724 e^{6 \pi}\right)\right. \\
& -625468) z^{5}+1764476 e^{2 \pi}\left(e ^ { 6 \pi } \left(61818120+35320311 e^{2 \pi}-60289650 e^{4 \pi}\right.\right. \\
& \left.\left.+256360 e^{6 \pi}\right)-37105141\right) \rho z^{4}-500055 e^{2 \pi}\left(-41423181-3714436 e^{4 \pi}\right. \\
& \left.+16341616 e^{6 \pi}+19305793 e^{8 \pi}+9490208 e^{10 \pi}\right) \rho^{2} z^{3}-3216040 e^{5 \pi} \rho^{3} z^{2} \\
& \sinh (\pi)(153644891-120359184 \sinh (2 \pi)+31576438 \cosh (2 \pi)) \\
& \left.-204805250 e^{2 \pi}\left(-784488+784488 e^{2 \pi}+61013 \pi\right) \rho^{4} z\right)+(-2403375 \\
& \left(e^{2 \pi}-1\right) \rho^{3}+\left(-13030+21489 e^{4 \pi}-4988 e^{6 \pi}-4147 e^{8 \pi}+676 e^{10 \pi}\right) z^{3} \\
& 357 e^{2 \pi}-986 e^{5 \pi} \rho z^{2} \sinh (\pi)(19421-3834 \sinh (2 \pi)+12388 \cosh (2 \pi)) \\
& \left.\left.+11310 e^{2 \pi}\left(-1627+1627 e^{2 \pi}-6375 \pi\right) \rho^{2} z\right) \frac{a 2}{9613500 \rho^{2}}\right) \cdot
\end{aligned}
$$

Consider the graph $\mathcal{Z}=\left\{\mathbf{z}_{\alpha}=(\alpha, \beta(\alpha)): \beta(\alpha)=0\right.$ and $\left.\alpha>0\right\}$. For all $\alpha>0$ the function $\mathbf{g}_{0}\left(\mathbf{z}_{\alpha}\right)=(0,0)$ then taking $r=0$ in Theorem 3 we compute the bifurcation functions $f_{1}(\alpha)=0$, and $f_{2}(\alpha)=\frac{\alpha}{500}\left(10\left(71 e^{2 \pi}\left(e^{2 \pi}-2\right)+95 \pi+\right.\right.$ 71) $\left.a_{2}-\left(284 e^{2 \pi}\left(e^{2 \pi}-2\right)-5 \pi+284\right) \alpha^{2}\right)$. 
For $\alpha^{*}=\sqrt{\frac{5 a_{2}}{2}\left(1+\frac{385 \pi}{284 e^{2 \pi}\left(e^{2 \pi}-2\right)-5 \pi+284}\right)}$ we have $f_{2}\left(\alpha^{*}\right)=0$ and $D f_{2}\left(\alpha^{*}\right) \neq 0$. Thus applying statement $(b)$ of Theorem 3 we have that system (25) has a periodic solution bifurcating from point $\mathbf{z}_{\alpha^{*}}$. Consequently, going back through the change of variables we have the existence of a periodic solution to system (11).

Proof of Theorem 2 statement $(i i)$. First we translate the point $(-7,-7,0)$ to the origin of coordinates. Then we use the change of variables $(x, y, z)=\varepsilon(8 X+$ $Z, X+\sqrt{7} Y-Z / 7,-8(\sqrt{7} Y+Z))$ the differential system (12) writes

$$
\begin{aligned}
\dot{X}= & -\sqrt{7} Y+\frac{\varepsilon}{568}\left(7 a_{2}-4\left(336 X^{2}-98 \sqrt{7} X Y+128 X Z+98 Y^{2}-2 \sqrt{7} Y Z\right.\right. \\
& \left.\left.+7 Z^{2}\right)\right), \\
\dot{Y}= & \sqrt{7} X+\frac{\varepsilon}{3976 \sqrt{7}}\left(6 0 \left(336 X^{2}-98 \sqrt{7} X Y+128 X Z+98 Y^{2}-2 \sqrt{7} Y Z\right.\right. \\
& \left.\left.+7 Z^{2}\right)-105 a_{2}\right), \\
\dot{Z}= & -8 Z+\frac{\varepsilon}{71}\left(4\left(336 X^{2}-98 \sqrt{7} X Y+128 X Z+98 Y^{2}-2 \sqrt{7} Y Z+7 Z^{2}\right)\right. \\
& \left.-7 a_{2}\right) .
\end{aligned}
$$

Using the cylindrical change of variables $(X, Y, Z)=(\rho \cos \theta, \rho \sin \theta, z)$ where $\rho>0$, system (26) becomes

$$
\begin{aligned}
\dot{\rho}= & \frac{\varepsilon}{27832}(4 \rho z(1009 \sqrt{7} \sin (2 \theta)-3031 \cos (2 \theta)-3241) \\
& +28 \rho^{2}(\sqrt{7}(509 \sin \theta+299 \sin (3 \theta))-2303 \cos \theta-49 \cos (3 \theta)) \\
& \left.+7\left(a_{2}-4 z^{2}\right)(49 \cos \theta-15 \sqrt{7} \sin \theta)\right), \\
\dot{\theta}= & \sqrt{7}-\frac{\varepsilon}{27832 \rho}(49 \sin \theta+15 \sqrt{7} \cos \theta)\left(7\left(a_{2}-4\left(31 \rho^{2}+z^{2}\right)\right)-476 \rho^{2} \cos (2 \theta)\right. \\
& -512 \rho z \cos \theta+8 \sqrt{7} \rho \sin \theta(49 \rho \cos \theta+z)), \\
\dot{z}= & -8 z+\frac{\varepsilon}{71}\left(-7 a_{2}+476 \rho^{2} \cos (2 \theta)+868 \rho^{2}+28 z^{2}+512 \rho z \cos \theta\right. \\
& -8 \sqrt{7} \rho \sin \theta(49 \rho \cos \theta+z)) .
\end{aligned}
$$

This differential system can be reduced to the normal form for applying the averaging theory. Taking $\theta$ as the new independent variable we obtain the differential system

$$
\begin{aligned}
\rho^{\prime}= & \frac{\varepsilon}{27832 \sqrt{7}}\left(28 \rho^{2}(\sqrt{7}(509 \sin \theta+299 \sin (3 \theta))-2303 \cos \theta-49 \cos (3 \theta))\right. \\
& +4 \rho z(1009 \sqrt{7} \sin (2 \theta)-3031 \cos (2 \theta)-3241)+7\left(a_{2}-4 z^{2}\right)
\end{aligned}
$$




$$
\begin{aligned}
& (49 \cos \theta-15 \sqrt{7} \sin \theta))+\mathcal{O}\left(\varepsilon^{2}\right), \\
z^{\prime}= & -\frac{8 z}{\sqrt{7}}-\frac{\varepsilon}{24353 \rho}\left(( 4 9 ( \sqrt { 7 } \rho + z \operatorname { s i n } \theta ) + 1 5 \sqrt { 7 } z \operatorname { c o s } \theta ) \left(a_{2}-4\left(31 \rho^{2}+z^{2}\right)\right.\right. \\
& \left.7-476 \rho^{2} \cos (2 \theta)-512 \rho z \cos \theta+8 \sqrt{7} \rho \sin \theta(49 \rho \cos \theta+z)\right)+\mathcal{O}\left(\varepsilon^{2}\right),
\end{aligned}
$$

here the derivatives are taken with respect to $\theta$. Using (19) we write the functions

$$
\begin{aligned}
\mathbf{g}_{0}(\mathbf{z})= & \left(0,\left(1-e^{16 \pi / \sqrt{7}}\right) z\right) \\
\mathbf{g}_{1}(\mathbf{z})= & \left(\frac{97\left(e^{-\frac{32 \pi}{\sqrt{7}}}-1\right) z^{2}}{37346}-\frac{13541\left(1-e^{-\frac{16 \pi}{\sqrt{7}}}\right) \rho z}{182896}, \frac{e^{-\frac{48 \pi}{\sqrt{7}}}}{168355768 \rho}\right. \\
& \left(e^{\frac{16 \pi}{\sqrt{7}}}-1\right)\left(90209 e^{\frac{32 \pi}{\sqrt{7}}} \rho\left(4724 \rho^{2}-23 a_{2}\right)+1488928 z^{3}+8 e^{\frac{16 \pi}{\sqrt{7}}} z^{2}\right. \\
& (7509965 \rho+186116 z))) \\
\mathbf{g}_{2}(\mathbf{z})= & \left(H_{1}(\mathbf{z}), H_{2}(\mathbf{z})\right) .
\end{aligned}
$$

where the functions $H_{1}$ and $H_{2}$ are provided in the appendix.

Consider the graph $\mathcal{Z}=\left\{\mathbf{z}_{\alpha}=(\alpha, \beta(\alpha)): \beta(\alpha)=0\right.$ and $\left.\alpha>0\right\}$. For all $\alpha>$ 0 the function $\mathbf{g}_{0}\left(\mathbf{z}_{\alpha}\right)=(0,0)$ then taking $r=0$ in Theorem 3 we compute the bifurcation functions $f_{1}(\alpha)=0$, and

$$
\begin{aligned}
f_{2}(\alpha)= & \frac{e^{-\frac{32 \pi}{\sqrt{7}}} \alpha}{2389353344}\left(189574 e^{\frac{16 \pi}{\sqrt{7}}}\left(23 a_{2}-4724 \alpha^{2}\right)-94787\left(23 a_{2}-4724 \alpha^{2}\right)\right. \\
& \left.+e^{\frac{32 \pi}{\sqrt{7}}}\left(26128 \sqrt{7} \pi\left(23 a_{2}-17684 \alpha^{2}\right)-94787\left(23 a_{2}-4724 \alpha^{2}\right)\right)\right) .
\end{aligned}
$$

The bifurcation function $f_{2}$ has the positive solution

$$
\begin{aligned}
\alpha^{*} & =\frac{1}{2} \sqrt{\frac{23 a_{2}\left(e^{\frac{32 \pi}{\sqrt{7}}}(26128 \sqrt{7} \pi-94787)+189574 e^{\frac{16 \pi}{\sqrt{7}}}-94787\right)}{e^{\frac{32 \pi}{\sqrt{7}}}(115511888 \sqrt{7} \pi-111943447)+223886894 e^{\frac{16 \pi}{\sqrt{7}}}-111943447}} \\
& \approx 0.0288042 \sqrt{a_{2}},
\end{aligned}
$$

such that $D f_{2}\left(\alpha^{*}\right) \approx-0.002 a_{2} \neq 0$. Thus applying statement $(b)$ of Theorem 3 we have that system (27) has a periodic solution bifurcating from point $\mathbf{z}_{\alpha}^{*}$. Consequently, going back through the change of variables we have the existence of a periodic solution to system (12).

Proof of Theorem 2 statement (iii). After translating the point $(-5 / 4,0,5 / 4)$ to the origin of coordinates we use the change of variables $(x, y, z)=\varepsilon(Y+$ $\left.Z, \frac{5 Z}{4}-\sqrt{2} X, Y-\frac{41 Z}{16}\right)$, then the differential system (13) writes

$$
\dot{X}=-\sqrt{2} Y+\frac{\varepsilon\left(320 a_{2}+12 \sqrt{2} X(32 Y+13 Z)-320 Y^{2}+20 Y Z+625 Z^{2}\right)}{912 \sqrt{2}},
$$




$$
\begin{aligned}
& \dot{Y}=\sqrt{2} X+\frac{\varepsilon\left(-320 a_{2}-12 \sqrt{2} X(32 Y+13 Z)+5\left(64 Y^{2}-4 Y Z-125 Z^{2}\right)\right)}{1140}, \\
& \dot{Z}=-\frac{5 Z}{4}+\frac{\varepsilon\left(320 a_{2}+12 \sqrt{2} X(32 Y+13 Z)-320 Y^{2}+20 Y Z+625 Z^{2}\right)}{1140} .
\end{aligned}
$$

Using the cylindrical change of variables $(X, Y, Z)=(\rho \cos \theta, \rho \sin \theta, z)$ where $\rho>0$, system (28) becomes

$$
\begin{aligned}
\dot{\rho}= & \frac{\varepsilon}{9120}\left(5\left(64 a_{2}+125 z^{2}\right)(5 \sqrt{2} \cos \theta-8 \sin \theta)+32 \rho^{2} \sin \theta(-73 \sqrt{2} \sin (2 \theta)\right. \\
& +20 \cos (2 \theta)+100)+2 \rho z(-287 \sqrt{2} \sin (2 \theta)+430 \cos (2 \theta)+350)), \\
\dot{\theta}= & \sqrt{2}+\frac{\varepsilon}{9120 \rho}(5 \sqrt{2} \sin \theta+8 \cos \theta)\left(320 a_{2}+625 z^{2}+4 \rho(5 \sin \theta(z-16 \rho \sin \theta)\right. \\
& +3 \sqrt{2} \cos \theta(32 \rho \sin \theta+13 z))), \\
\dot{z}= & -\frac{5 z}{4}+\frac{\varepsilon}{1140}(4 \rho(5 \sin \theta(z-16 \rho \sin \theta)+3 \sqrt{2} \cos \theta(32 \rho \sin \theta+13 z)) \\
& \left.+320 a_{2}+625 z^{2}\right) .
\end{aligned}
$$

This differential system can be reduced to the normal form for applying the averaging theory. Taking $\theta$ as the new independent variable we obtain the differential system

$$
\begin{aligned}
\rho^{\prime}= & \frac{\varepsilon}{9120 \sqrt{2}}\left(-2560 a_{2} \sin \theta+\sqrt{2} \cos \theta\left(1600 a_{2}+3125 \rho^{4} z^{2}-1148 \rho^{3} z \sin \theta\right.\right. \\
& \left.-1168 \rho^{2}\right)+700 \rho^{3} z+4 \rho^{2}\left(292 \sqrt{2} \cos (3 \theta)+50 \sin \theta\left(16-25 \rho^{2} z^{2}\right)\right. \\
& +5 \cos (2 \theta)(32 \sin \theta+43 \rho z)))+\frac{\varepsilon^{2}}{83174400 \sqrt{2} \rho}\left(-2560 a_{2} \sin \theta+\sqrt{2} \cos \theta\right. \\
& \left(1600 a_{2}+3125 \rho^{4} z^{2}-1148 \rho^{3} z \sin \theta-1168 \rho^{2}\right)+700 \rho^{3} z+4 \rho^{2} \\
& \left.\left(292 \sqrt{2} \cos (3 \theta)+50 \sin \theta\left(16-25 \rho^{2} z^{2}\right)+5 \cos (2 \theta)(32 \sin \theta+43 \rho z)\right)\right) \\
& +\left(\sin \theta\left(1600 a_{2}+3125 \rho^{4} z^{2}+2336 \rho^{2} \cos (2 \theta)+736 \rho^{2}\right)+20 \sqrt{2} \cos \theta\right. \\
& \left.\left(64 a_{2}+125 \rho^{4} z^{2}+\rho^{2} \sin \theta(32 \sin \theta+43 \rho z)\right)+2 \rho^{3} z(287 \cos (2 \theta)+337)\right) \\
& +\mathcal{O}\left(\varepsilon^{3}\right), \\
z^{\prime}= & -\frac{5 z}{4 \sqrt{2}}+\frac{\varepsilon 1024}{36480 \sqrt{2} \rho^{2}}\left(\left(10 a_{2}+\rho^{2}(6 \sqrt{2} \sin (2 \theta)-5)+5 \rho^{2} \cos (2 \theta)\right)-16 \rho z\right. \\
& \left(-780 a_{2} \sin \theta+6 \sqrt{2}\left(200 a_{2}-141 \rho^{2}\right) \cos \theta+\rho^{2}(1265 \sin \theta+525 \sin (3 \theta)\right. \\
& +534 \sqrt{2} \cos (3 \theta)))+1875 \rho^{5} z^{3}(13 \sin \theta-20 \sqrt{2} \cos \theta)+2 \rho^{4} z^{2} \\
& (1221 \sqrt{2} \sin (2 \theta)-4875 \cos (2 \theta)+5515)+\frac{\varepsilon^{2}}{665395200 \rho^{3}}\left(\left(\operatorname { s i n } \theta \left(1600 a_{2}\right.\right.\right. \\
&
\end{aligned}
$$




$$
\begin{aligned}
& \left.+3125 \rho^{4} z^{2}+2336 \rho^{2} \cos (2 \theta)+736 \rho^{2}\right)+20 \sqrt{2} \cos \theta\left(64 a_{2}+125 \rho^{4} z^{2}\right. \\
& \left.\left.+\rho^{2} \sin \theta(32 \sin \theta+43 \rho z)\right)+2 \rho^{3} z(287 \cos (2 \theta)+337)\right)(\rho(5 \sqrt{2} z \sin \theta \\
& \left(2496 a_{2}+4875 \rho^{4} z^{2}-4048 \rho^{2}\right)-24 z \cos \theta\left(1600 a_{2}+3125 \rho^{4} z^{2}-1128 \rho^{2}\right) \\
& +12 \rho \sin (2 \theta)\left(407 \rho^{2} z^{2}+1024\right)+10 \sqrt{2} \rho \cos (2 \theta)\left(512-975 \rho^{2} z^{2}\right) \\
& \left.-8400 \sqrt{2} \rho^{2} z \sin (3 \theta)-17088 \rho^{2} z \cos (3 \theta)\right)+10 \sqrt{2}\left(1024 a_{2}+1103 \rho^{4} z^{2}\right. \\
& \left.\left.\left.-512 \rho^{2}\right)\right)\right)+\mathcal{O}\left(\varepsilon^{3}\right),
\end{aligned}
$$

here the derivatives are taken with respect to $\theta$. Using (19) we write the functions

$$
\begin{aligned}
\mathbf{g}_{0}(\mathbf{z})= & \left(0,\left(1-e^{\frac{5 \pi}{2 \sqrt{2}}}\right) z\right) \\
\mathbf{g}_{1}(\mathbf{z})= & \left(\frac{\rho^{3} z\left(-95625 e^{-\frac{5 \pi}{\sqrt{2}}} \sqrt{2} \rho z+95625 \sqrt{2} \rho z-121264 e^{-\frac{5 \pi}{2 \sqrt{2}}}+121264\right)}{2558160},\right. \\
& \frac{e^{-\frac{15 \pi}{2 \sqrt{2}}}}{15348960 \rho^{2}}\left(e^{\frac{5 \pi}{2 \sqrt{2}}}-1\right)\left(22528 e^{\frac{5 \pi}{\sqrt{2}}}\left(153 a_{2}-112 \rho^{2}\right)-3538125 \sqrt{2} \rho^{5} z^{3}\right. \\
& \left.\left.-15 e^{\frac{5 \pi}{2 \sqrt{2}}} \rho^{4} z^{2}(235875 \sqrt{2} \rho z-240416)\right)\right) \\
\mathbf{g}_{2}(\mathbf{z})= & \left(I_{1}(\mathbf{z}), I_{2}(\mathbf{z})\right) .
\end{aligned}
$$

where the functions $I_{1}$ and $I_{2}$ are provided in the appendix. Consider the graph $\mathcal{Z}=\left\{\mathbf{z}_{\alpha}=(\alpha, \beta(\alpha)): \beta(\alpha)=0\right.$ and $\left.\alpha>0\right\}$. For all $\alpha>0$ the function $\mathbf{g}_{0}\left(\mathbf{z}_{\alpha}\right)=(0,0)$ then taking $r=0$ in Theorem 3 we compute the bifurcation functions $f_{1}(\alpha)=0$, and

$$
\begin{aligned}
f_{2}(\alpha)= & \frac{e^{-\frac{5 \pi}{2 \sqrt{2}}}}{633798675}\left(44096\left(153 \alpha a_{2}-112 \alpha^{3}\right)+8 e^{\frac{5 \pi}{2 \sqrt{2}}} \alpha\left(5512\left(112 \alpha^{2}-153 a_{2}\right)\right.\right. \\
& \left.\left.+14535 \pi \sqrt{2}\left(306 a_{2}-131 \alpha^{2}\right)\right)\right) .
\end{aligned}
$$

The bifurcation function $f_{2}$ has the positive solution

$$
\alpha^{*}=3 \sqrt{\frac{34 a_{2}\left(e^{\frac{5 \pi}{2 \sqrt{2}}}(14535 \sqrt{2} \pi-2756)+2756\right)}{e^{\frac{5 \pi}{2 \sqrt{2}}}(1904085 \sqrt{2} \pi-617344)+617344}},
$$

such that $D f_{2}\left(\alpha^{*}\right) \approx-0.47 a_{2} \neq 0$. Thus applying statement $(b)$ of Theorem 3 we have that system (29) has a periodic solution bifurcating from point $\mathbf{z}_{\alpha}^{*}$. Consequently, going back through the change of variables we have the existence of a periodic solution to system (13). 
Proof of Theorem 2 statement $(i v)$. First we translate the point $(-5,0,0)$ to the origin of coordinates, then using the change of variables $(x, y, z)=\varepsilon\left(-\frac{X}{\sqrt{5}}+\right.$ $\left.\frac{Y}{\sqrt{5}}-\frac{Z}{2}, X+Y+Z, \sqrt{5} X-\sqrt{5} Y-2 Z\right)$ the differential system (14) writes

$$
\begin{aligned}
\dot{X}= & -\sqrt{5} Y-\varepsilon \frac{(2 \sqrt{5}+5)}{1800 \sqrt{5}}\left(20(\sqrt{5}+6) X^{2}-100 a_{2}-20(\sqrt{5}-6) Y^{2}\right. \\
& \left.+2 X(80 Y+125 Z+2 \sqrt{5} Z)+(250-4 \sqrt{5}) Y Z+85 Z^{2}\right) \\
\dot{Y}= & \sqrt{5} X-\varepsilon \frac{(2 \sqrt{5}-5)}{1800 \sqrt{5}}\left(20(\sqrt{5}+6) X^{2}-100 a_{2}-20(\sqrt{5}-6) Y^{2}\right. \\
& \left.+2 X(80 Y+125 Z+2 \sqrt{5} Z)+(250-4 \sqrt{5}) Y Z+85 Z^{2}\right) \\
\dot{Z}= & -2 Z+\frac{1}{450} \varepsilon\left(20(\sqrt{5}+6) X^{2}-100 a_{2}+2 X(80 Y+125 Z+2 \sqrt{5} Z)\right. \\
& \left.-20(\sqrt{5}-6) Y^{2}+(250-4 \sqrt{5}) Y Z+85 Z^{2}\right) .
\end{aligned}
$$

Using the cylindrical change of variables $(X, Y, Z)=(\rho \cos \theta, \rho \sin \theta, z)$ where $\rho>0$, system (30) becomes

$$
\begin{aligned}
\dot{\rho}= & \frac{\varepsilon}{1800}\left(5\left(20 a_{2}-17 z^{2}\right)((\sqrt{5}+2) \cos \theta-(\sqrt{5}-2) \sin \theta)\right. \\
& -2 \sqrt{5} \rho^{2}(3(\sqrt{5}+10) \sin (3 \theta)+(37 \sqrt{5}-50) \sin \theta-3(\sqrt{5}-10) \cos (3 \theta) \\
& +(37 \sqrt{5}+50) \cos \theta)-2 \rho z(240 \sin (2 \theta)+129 \sqrt{5} \cos (2 \theta)+260)), \\
\dot{\theta}= & \sqrt{5}-\frac{\varepsilon}{1800 \sqrt{5} \rho}((2 \sqrt{5}-5) \cos \theta-(2 \sqrt{5}+5) \sin \theta)\left(120 \rho^{2}-100 a_{2}\right. \\
& +2 \rho(10 \sqrt{5} \rho \cos (2 \theta)+\cos \theta(80 \rho \sin \theta+(2 \sqrt{5}+125) z) \\
& \left.+(125-2 \sqrt{5}) z \sin \theta)+85 z^{2}\right), \\
\dot{z}= & -2 z+\frac{\varepsilon}{450}\left(-100 a_{2}+120 \rho^{2}+2 \rho(10 \sqrt{5} \rho \cos (2 \theta)+\cos \theta(80 \rho \sin \theta\right. \\
& \left.+(2 \sqrt{5}+125) z)+(125-2 \sqrt{5}) z \sin \theta)+85 z^{2}\right) .
\end{aligned}
$$

This differential system can be reduced to the normal form for applying the averaging theory. Taking $\theta$ as the new independent variable we obtain the differential system

$$
\begin{aligned}
\rho^{\prime}= & \frac{\varepsilon}{1800 \sqrt{5}}\left(5\left(20 a_{2}-17 z^{2}\right)((\sqrt{5}+2) \cos \theta-(\sqrt{5}-2) \sin \theta)-2 \sqrt{5} \rho^{2}\right. \\
& (3(\sqrt{5}+10) \sin (3 \theta)+(37 \sqrt{5}-50) \sin \theta-3(\sqrt{5}-10) \cos (3 \theta)+\cos \theta
\end{aligned}
$$




$$
\begin{aligned}
& (37 \sqrt{5}+50))-2 \rho z(240 \sin (2 \theta)+129 \sqrt{5} \cos (2 \theta)+260))+\mathcal{O}\left(\varepsilon^{3}\right), \\
z^{\prime}= & -\frac{2 \sqrt{5} z}{25}+\frac{\varepsilon}{4500 \sqrt{5} \rho}(10 \rho+(2 \sqrt{5}+5) z \sin \theta+(5-2 \sqrt{5}) z \cos \theta)\left(120 \rho^{2}\right. \\
& -100 a_{2}+85 z^{2}+2 \rho(10 \sqrt{5} \rho \cos (2 \theta)+\cos \theta(80 \rho \sin \theta+(2 \sqrt{5}+125) z) \\
& +(125-2 \sqrt{5}) z \sin \theta))-\varepsilon^{2} \frac{((2 \sqrt{5}-5) \cos \theta-(2 \sqrt{5}+5) \sin \theta)}{40500000 \sqrt{5} \rho^{2}}(-10 \rho \\
& -(2 \sqrt{5}+5) z \sin \theta+(2 \sqrt{5}-5) z \cos \theta)\left(120 \rho^{2}-100 a_{2}+85 z^{2}+z \sin \theta\right. \\
& (125-2 \sqrt{5})+2 \rho(10 \sqrt{5} \rho \cos (2 \theta)+\cos \theta(80 \rho \sin \theta+(2 \sqrt{5}+125) z)))^{2} \\
& +\mathcal{O}\left(\varepsilon^{3}\right),
\end{aligned}
$$

here the derivatives are taken with respect to $\theta$. Using (19) we write the functions

$$
\begin{aligned}
\mathbf{g}_{0}(\mathbf{z})= & \left(0,\left(1-e^{\frac{4 \pi}{\sqrt{5}}}\right) z\right), \\
\mathbf{g}_{1}(\mathbf{z})= & \left(\frac { e ^ { - \frac { 1 2 \pi } { \sqrt { 5 } } } } { 1 2 6 0 0 \rho } ( 1 - e ^ { \frac { 4 \pi } { \sqrt { 5 } } } ) \left(140 e^{\frac{8 \pi}{\sqrt{5}}} \rho\left(10 a_{2}+(\sqrt{5}-12) \rho^{2}\right)+34\left(1+e^{\frac{4 \pi}{\sqrt{5}}}\right)\right.\right. \\
& \left.(\sqrt{5}-10) z^{3}-7(27 \sqrt{5}+412) e^{\frac{4 \pi}{\sqrt{5}}} \rho z^{2}\right)-\frac{e^{-\frac{8 \pi}{\sqrt{5}}}}{25200}\left(e^{\frac{4 \pi}{\sqrt{5}}}-1\right) z \\
& \left.\left(7 e^{\frac{4 \pi}{\sqrt{5}}}(123 \sqrt{5}+520) \rho+170\left(1+e^{\frac{4 \pi}{\sqrt{5}}}\right)(2 \sqrt{5}+1) z\right)\right), \\
\mathbf{g}_{2}(\mathbf{z})= & \left(J_{1}(\mathbf{z}), J_{2}(\mathbf{z})\right) .
\end{aligned}
$$

where the functions $J_{1}$ and $J_{2}$ are provided in the appendix.

Consider the graph $\mathcal{Z}=\left\{\mathbf{z}_{\alpha}=(\alpha, \beta(\alpha)): \beta(\alpha)=0\right.$ and $\left.\alpha>0\right\}$. For all $\alpha>$ 0 the function $\mathbf{g}_{0}\left(\mathbf{z}_{\alpha}\right)=(0,0)$, then taking $r=0$ in Theorem 3 we compute the bifurcation functions $f_{1}(\alpha)=0$, and

$$
\begin{aligned}
f_{2}(\alpha)= & \frac{e^{-\frac{8 \pi}{\sqrt{5}}} \alpha}{324000}\left((956 \sqrt{5}+5625) \alpha^{2}+2 e^{\frac{4 \pi}{\sqrt{5}}}\left(10(123 \sqrt{5}+520) a_{2}-\right.\right. \\
& \left.(956 \sqrt{5}+5625) \alpha^{2}\right)+e^{\frac{8 \pi}{\sqrt{5}}}\left((956 \sqrt{5}+5625) \alpha^{2}+180 \pi \sqrt{5}\left(8 a_{2}-19 \alpha^{2}\right)\right. \\
& \left.\left.-10(123 \sqrt{5}+520) a_{2}\right)-10(123 \sqrt{5}+520) a_{2}\right) .
\end{aligned}
$$

The bifurcation function $f_{2}$ has the positive solution $\alpha^{*}=$

$$
\begin{aligned}
& \sqrt{\frac{10 a_{2}\left(e^{\frac{8 \pi}{\sqrt{5}}}(144 \pi \sqrt{5}-123 \sqrt{5}-520)-123 \sqrt{5}+2 e^{\frac{4 \pi}{\sqrt{5}}}(123 \sqrt{5}+520)-520\right)}{e^{\frac{8 \pi}{\sqrt{5}}}(3420 \pi \sqrt{5}-956 \sqrt{5}-5625)-956 \sqrt{5}+2 e^{\frac{4 \pi}{\sqrt{5}}}(956 \sqrt{5}+5625)-5625}} \\
& \approx 0.369082 \sqrt{a_{2}},
\end{aligned}
$$


such that $D f_{2}\left(\alpha^{*}\right) \approx-0.01 a_{2} \neq 0$. Thus applying statement $(b)$ of Theorem 3 we have that system (27) has a periodic solution bifurcating from point $\mathbf{z}_{\alpha}^{*}$. Consequently, going back through the change of variables we have the existence of a periodic solution to system (12).

Proof of Theorem 2 statement $(v)$. First we translate the equilibrium point $(4 / 5,4 / 5,4 / 5)$ to the origin. Then using the change of variables $(x, y, z)=$ $\varepsilon(Z-2 Y, \sqrt{3} X+Y+Z Y,(-\sqrt{3}) X+Y+Z)$ the differential system (15) writes

$$
\begin{aligned}
& \dot{X}=-\frac{1}{5}(4 \sqrt{3} Y)+\frac{\varepsilon\left(3 X^{2}-2 \sqrt{3} X(Y-2 Z)-3 Y(Y+4 Z)\right)}{4 \sqrt{3}}, \\
& \dot{Y}=\frac{4 \sqrt{3} X}{5}+\frac{1}{4} \varepsilon\left(-X^{2}-2 \sqrt{3} X(Y-2 Z)+Y(Y+4 Z)\right) \\
& \dot{Z}=\varepsilon\left(a_{2}-2\left(X^{2}+Y^{2}\right)+\frac{Z^{2}}{2}\right) .
\end{aligned}
$$

Using the cylindrical change of variables $(X, Y, Z)=(\rho \cos \theta, \rho \sin \theta, z)$ where $\rho>0$, system (31) becomes

$$
\begin{aligned}
& \dot{\rho}=\frac{\varepsilon}{4} \rho(-\rho \sin (3 \theta)+\sqrt{3} \rho \cos (3 \theta)+4 z), \\
& \dot{\theta}=\frac{4 \sqrt{3}}{5}+\frac{\varepsilon}{4}(\sqrt{3}(4 z-\rho \sin (3 \theta))-\rho \cos (3 \theta)), \\
& \dot{z}=\varepsilon\left(a_{2}-2 \rho^{2}+\frac{z^{2}}{2}\right) .
\end{aligned}
$$

This differential system can be reduced to the normal form for applying the averaging theory. Taking $\theta$ as the new independent variable we obtain the differential system

$$
\begin{aligned}
& \rho^{\prime}=\frac{5 \rho \varepsilon(-\rho \sin (3 \theta)+\sqrt{3} \rho \cos (3 \theta)+4 z)}{16 \sqrt{3}}+\mathcal{O}\left(\varepsilon^{2}\right), \\
& z^{\prime}=\frac{5 \varepsilon\left(2 a_{2}-4 \rho^{2}+z^{2}\right)}{8 \sqrt{3}}+\mathcal{O}\left(\varepsilon^{2}\right),
\end{aligned}
$$

here the derivatives are taken with respect to $\theta$. Using (19) we write the functions $\mathbf{g}_{0} \equiv 0$ and $\mathbf{g}_{1}(\mathbf{z})=\pi\left(\frac{5 r z}{2 \sqrt{3}}, \frac{5\left(2 a_{2}-4 r^{2}+z^{2}\right)}{4 \sqrt{3}}\right)$. The averaged function $\mathbf{g}_{1}$ has the solutions $\mathbf{z}_{ \pm}= \pm\left(\omega \sqrt{\frac{a_{2}}{2}}, 0\right)$. The result follows by taking $r=0$ and $\mathbf{z}^{*}=\mathbf{z}_{+}$and applying Corollary 4. The eigenvalues of $D \mathbf{g}_{1}\left(\mathbf{z}_{+}\right)$are $\pm i 5 \sqrt{\frac{a_{2}}{6}}$.

Proof of Theorem 2 statement (vi). First we translate to the origin of coordinates the point $(-10 / 43,-10 / 43,-10 / 43)$. Then using the change of variables 
$(x, y, z)=\varepsilon\left(X+Z,-\frac{X}{2}+\frac{\sqrt{3} Y}{2}+Z,-\frac{X}{2}-\frac{\sqrt{3} Y}{2}+Z\right)$ the differential system

(16) writes

$$
\begin{aligned}
& \dot{X}=-\sqrt{3} Y+\frac{\varepsilon}{40}\left(-13 X^{2}+26 \sqrt{3} X Y+86 X Z+13 Y^{2}+86 \sqrt{3} Y Z\right), \\
& \dot{Y}=\sqrt{3} X+\frac{\varepsilon}{40}\left(13 \sqrt{3} X^{2}+26 X Y-86 \sqrt{3} X Z-13 \sqrt{3} Y^{2}+86 Y Z\right), \\
& \dot{Z}=-\frac{69}{43} Z+\frac{\varepsilon}{40}\left(40 a_{2}-43\left(X^{2}+Y^{2}\right)+52 Z^{2}\right) .
\end{aligned}
$$

Using the cylindrical change of variables $(X, Y, Z)=(\rho \cos \theta, \rho \sin \theta, z)$ where $\rho>0$, system (32) becomes

$$
\begin{aligned}
& \dot{\rho}=\varepsilon \frac{\rho}{40}(13 \sqrt{3} \rho \sin (3 \theta)-13 \rho \cos (3 \theta)+86 z), \\
& \dot{\theta}=\sqrt{3}+\frac{\varepsilon}{40}(13 \rho(\sin (3 \theta)+\sqrt{3} \cos (3 \theta))-86 \sqrt{3} z), \\
& \dot{z}=-\frac{69}{43} z+\varepsilon\left(a_{2}+\frac{1}{40}\left(52 z^{2}-43 \rho^{2}\right)\right) .
\end{aligned}
$$

This differential system can be reduced to the normal form for applying the averaging theory. Taking $\theta$ as the new independent variable we obtain the differential system

$$
\begin{aligned}
\rho^{\prime}= & \frac{\varepsilon \rho}{40 \sqrt{3}}(13 \sqrt{3} \rho \sin (3 \theta)-13 \rho \cos (3 \theta)+86 z)+\frac{\varepsilon^{2} \rho}{4800}(13 \rho(\cos (3 \theta) \\
& -\sqrt{3} \sin (3 \theta))-86 z)(13 \rho(\sin (3 \theta)+\sqrt{3} \cos (3 \theta))-86 \sqrt{3} z)+\mathcal{O}\left(\varepsilon^{3}\right) \\
z^{\prime}= & -\frac{23 \sqrt{3}}{43} z+\frac{\varepsilon}{5160}\left(43 \sqrt{3}\left(40 a_{2}-43\left(\rho^{2}+2 z^{2}\right)\right)+897 \rho z(\sin (3 \theta)\right. \\
& +\sqrt{3} \cos (3 \theta)))+\frac{\varepsilon^{2}}{206400}\left(1 3 \rho \left(344\left(28 z^{2}-5 a_{2}\right)(\sin (3 \theta)+\sqrt{3} \cos (3 \theta))\right.\right. \\
& \left.+1849 \rho^{2}(\sin (3 \theta)+\sqrt{3} \cos (3 \theta))-299 \rho z(3 \sin (6 \theta)+\sqrt{3} \cos (6 \theta))\right) \\
& \left.-4 \sqrt{3} z\left(41697 \rho^{2}+79507 z^{2}-36980 a_{2}\right)\right)+\mathcal{O}\left(\varepsilon^{3}\right),
\end{aligned}
$$

here the derivatives are taken with respect to $\theta$. Using (19) we write the functions

$$
\begin{aligned}
\mathbf{g}_{0}(\mathbf{z})= & \left(0,\left(1-e^{\frac{46 \sqrt{3} \pi}{43}}\right) z\right) \\
\mathbf{g}_{1}(\mathbf{z})= & \left(\frac{1849}{1380}\left(1-e^{-\frac{1}{43}(46 \sqrt{3} \pi)}\right) \rho z,\left(e^{\frac{46 \sqrt{3} \pi}{43}}\left(40 a_{2}-43 \rho^{2}\right)-86 z^{2}\right)\right. \\
& \left.\left(e^{\frac{46 \sqrt{3} \pi}{43}}-1\right) \frac{43 e^{-\frac{1}{43}(92 \sqrt{3} \pi)}}{2760}\right), \\
\mathbf{g}_{2}(\mathbf{z})= & \left(\frac { 1 8 4 9 e ^ { - \frac { 1 } { 4 3 } ( 1 8 4 \sqrt { 3 } \pi ) } \rho } { 2 6 6 0 0 8 8 0 8 7 2 1 6 0 0 } \left(e ^ { \frac { 1 8 4 \sqrt { 3 } \pi } { 4 3 } } \left(1 1 6 4 0 0 9 7 \left(240(46 \sqrt{3} \pi-43) a_{2}\right.\right.\right.\right.
\end{aligned}
$$




$$
\begin{aligned}
& \left.\left.+19651 z^{2}\right)-3003145026(46 \sqrt{3} \pi-43) \rho^{2}+45396995982 \rho z\right) \\
& +22989 e^{\frac{138 \sqrt{3} \pi}{43}}\left(130634\left(40 a_{2}-43 \rho^{2}\right)-2456883 \rho z\right)+172180314824 \\
& \left.e^{\frac{46 \sqrt{3} \pi}{43}} z^{2}-64567618059 z^{2}+4557 e^{\frac{92 \sqrt{3} \pi}{43}} z(2432365 \rho-73810016 z)\right) \\
& \frac{e^{-\frac{1}{43}(230 \sqrt{3} \pi)}}{446414827786341650812800}\left(e ^ { \frac { 1 8 4 \sqrt { 3 } \pi } { 4 3 } } \left(-\left(60(391 \sqrt{3} \pi-1849) a_{2}\right.\right.\right. \\
& \left.+75809 z^{2}\right) 6719819923969996144 z+69703216719171814113816 \rho^{3} \\
& ++67860377243786125751610 \rho z^{2}-10446454479 \rho^{2} z \\
& (70137521867896 \sqrt{3} \pi-252393774165))-4245723465 e^{\frac{138 \sqrt{3} \pi}{43}} z \\
& \left(175587701015104 a_{2}+61893 \rho(10033543 \rho .+666198742 z)\right) \\
& -69703216719171814113816 e^{\frac{230 \sqrt{3} \pi}{43}} \rho^{3}+400704542021311862565756 z^{3} \\
& +1951360992537417972 e^{\frac{92 \sqrt{3} \pi}{43}} z^{2}(79833 \rho+329509 z) \\
& \left.\left.-336382138219685488 e^{\frac{46 \sqrt{3} \pi}{43}} z^{2}(144417 \rho+1588291 z)\right)\right) .
\end{aligned}
$$

Consider the graph $\mathcal{Z}=\left\{\mathbf{z}_{\alpha}=(\alpha, \beta(\alpha)): \beta(\alpha)=0\right.$ and $\left.\alpha>0\right\}$. For all $\alpha>0$ the function $\mathbf{g}_{0}\left(\mathbf{z}_{\alpha}\right)=(0,0)$, then taking $r=0$ in Theorem 3 we compute the bifurcation functions $f_{1}(\alpha)=0$, and

$$
f_{2}(\alpha)=\frac{1849 e^{-\frac{92 \sqrt{3} \pi}{43}}}{3808800}\left(e^{\frac{92 \sqrt{3} \pi}{43}}(46 \sqrt{3} \pi-43)+86 e^{\frac{46 \sqrt{3} \pi}{43}}-43\right) \alpha\left(40 a_{2}-43 \alpha^{2}\right) .
$$

For $\alpha^{*}=2 \sqrt{\frac{10 a_{2}}{43}}$ we have $f_{2}\left(\alpha^{*}\right)=0$ and $D f_{2}\left(\alpha^{*}\right) \neq 0$. Thus applying statement $(b)$ of Theorem 3 we have that system (33) has a periodic solution bifurcating from point $\mathbf{z}_{\alpha}^{*}$. Consequently, going back through the change of variables we have the existence of a periodic solution to system (16).

\section{Conclusions}

In this paper we use recent results in averaging theory (Theorem 3) for studying the zero-Hopf and the Hopf bifurcation of the sixteen 3-dimensional differential systems provided by Jafari et al [5] in 2013. These systems have equilibria only for a certain choice of the parameter $a$, and we show that under some conditions a periodic solution emerges in either a zero-Hopf or a Hopf bifurcation exhibits by the system and after the equilibria disappear. Moreover, we show graphically that the periodic orbit which is born in such bifurcations is the origin of a period doubling cascade which originates the chaotic motion in those differential systems.

Here we illustrated how the averaging theory is useful for studying the periodic orbits which bifurcate from a zero-Hopf or from a Hopf equilibrium point. 
The averaging method here presented does not provide information on the periodic solutions of system SNE 14 of [5], in the future we shall try to improve the averaging theory in order to apply it that differential system.

\section{Appendix}

Here we present the coordinate functions of $\mathbf{g}_{2}$ that appears in the proof of statements $(i i),(i i i)$ and $(i v)$ of Theorem 2.

$$
\begin{aligned}
& H_{1}(\mathbf{z})=-\frac{13541 a_{2} \rho}{14840704}+\frac{\pi a_{2} \rho}{568 \sqrt{7}}+e^{-\frac{32 \pi}{\sqrt{7}}}\left(\frac{899 a_{2} z^{2}}{40086032 \rho}-\frac{597507923 a_{2} z}{47083369573856}\right. \\
& \left.-\frac{703742848467097925 \rho z^{2}}{31898417885852553728}+\frac{3392361662905917 \rho^{2} z}{116684360646408632}\right)-\frac{189618713 a_{2} z^{2}}{10586680965168 \rho} \\
& +e^{-\frac{48 \pi}{\sqrt{7}}}\left(-\frac{42083 a_{2} z^{2}}{9319261413 \rho}-\frac{46840313877851 z^{3}}{38980159313748576}+\frac{1820956889005487 \rho z^{2}}{115614906197860608}\right) \\
& +e^{-\frac{16 \pi}{\sqrt{7}}}\left(\frac{13541 a_{2} \rho}{14840704}+\frac{4046213933 a_{2} z}{6557112770432}-\frac{15991921 \rho^{3}}{85334048}-\frac{23113407207573 \rho^{2} z}{195893744016656}\right) \\
& -\frac{47497703966367 a_{2} z}{78588870014419072}+\frac{15991921 \rho^{3}}{85334048}-\frac{4421 \pi \rho^{3}}{3266 \sqrt{7}}-\frac{16264 e^{-16 \sqrt{7} \pi} z^{4}}{1048694353 \rho} \\
& +\frac{247945373 z^{4}}{13482014602168 \rho}-\frac{19453239836 e^{-\frac{96 \pi}{\sqrt{7}}} z^{3}}{118452839157093}+\frac{45877191435540374951287 \rho z^{2}}{2993773813265605489598976} \\
& +\frac{19660203760820886413604205145 z^{3}}{21759296009409979593131149751776}+\frac{89976430564062929487 \rho^{2} z}{1011920113914411773456} \\
& +e^{-\frac{64 \pi}{\sqrt{7}}}\left(-\frac{1739 z^{4}}{74243848 \rho}+\frac{8088305746441 z^{3}}{6482324361591568}-\frac{112649333870939 \rho z^{2}}{12499380049315264}\right) \\
& +e^{-\frac{80 \pi}{\sqrt{7}}}\left(\frac{306340 z^{4}}{14913732967 \rho}-\frac{6272329328873 z^{3}}{7986095211566962}\right) \text {, } \\
& H_{2}(\mathbf{z})=e^{-\frac{32 \pi}{\sqrt{7}}}\left(-\frac{20409 a_{2}^{2} z}{806731394 \rho^{2}}-\frac{13593232675 a_{2} z^{2}}{5737473674128 \rho}+\frac{8622181081 a_{2} z}{2254007514836}\right. \\
& \left.+\frac{121148255304709 \rho z^{2}}{171407026014574}-\frac{9933070595407 \rho^{2} z}{207368691364912}\right)+e^{-\frac{16 \pi}{\sqrt{7}}}\left(\frac{20409 a_{2}^{2} z}{806731394 \rho^{2}}\right. \\
& +\frac{17031 a_{2} \rho}{927544}+\frac{12143784230186761 a_{2} z^{3}}{316096608568406160880 \rho^{2}}+\frac{354453085049431 a_{2} z^{2}}{207977542894816233 \rho} \\
& -\frac{8622181081 a_{2} z}{2254007514836}+\frac{97 \pi a_{2} z}{3479 \sqrt{7}}-\frac{54536545 \rho^{3}}{14724761}-\frac{24457919708879017 z^{5}}{595620109778216080404 \rho^{2}} \\
& -\frac{224198124665220307732284401468 z^{4}}{102793760349089735962832109971811 \rho} \\
& -\frac{116238165545186323672338583 z^{3}}{2690539476878912070359141040}-\frac{253261733610700641411573827 \rho z^{2}}{740098259404736722171720962}
\end{aligned}
$$




$$
\begin{aligned}
& \left.+\frac{9933070595407 \rho^{2} z}{207368691364912}-\frac{157357637 \pi \rho^{2} z}{17043621 \sqrt{7}}\right)-\frac{17031 a_{2} \rho}{927544} \\
& +e^{-\frac{48 \pi}{\sqrt{7}}}\left(-\frac{653124039 a_{2} z^{3}}{9704487615928 \rho^{2}}+\frac{4436646543253 a_{2} z^{2}}{3790211250695408 \rho}+\frac{269509153422410091 z^{3}}{3488889456265123064}\right. \\
& \left.-\frac{56593678003503976 \rho z^{2}}{102098815565607553}\right)+e^{-\frac{96 \pi}{\sqrt{7}}}\left(-\frac{1004634 a_{2} z^{3}}{132200451845 \rho^{2}}-\frac{9002928 z^{5}}{104396130769 \rho^{2}}\right. \\
& \left.+\frac{16223479764 z^{4}}{4620818852783 \rho}+\frac{42924541078 z^{3}}{14145448347415}\right)+e^{-\frac{80 \pi}{\sqrt{7}}}\left(-\frac{93459 a_{2} z^{3}}{39052264048 \rho^{2}}\right. \\
& \left.+\frac{15516419 z^{5}}{267908925508 \rho^{2}}-\frac{70253877690368467 z^{4}}{12331001516837560228 \rho}+\frac{1348260373986075 z^{3}}{21873915086301712}\right) \\
& +e^{-\frac{64 \pi}{\sqrt{7}}}\left(\frac{222327526 a_{2} z^{3}}{5718920087111 \rho^{2}}-\frac{227003953 a_{2} z^{2}}{448946020761 \rho}+\frac{65444853665 z^{4}}{19753624913484 \rho}\right. \\
& \left.-\frac{109952417460403592 z^{3}}{1113807488504463291}+\frac{703608436890712 \rho z^{2}}{3708743077506621}\right)+\frac{54536545 \rho^{3}}{14724761} \\
& +\frac{3232536 e^{-\frac{128 \pi}{\sqrt{7}}} z^{5}}{51386023297 \rho^{2}}+e^{-16 \sqrt{7} \pi}\left(\frac{210277 z^{5}}{32463122538 \rho^{2}}+\frac{874257394784 z^{4}}{829169874099651 \rho}\right) \\
& I_{1}(\mathbf{z})=a_{2}\left(\frac{16(14535 \sqrt{2} \pi-2756) \rho}{4142475}+\frac{2310379625 \rho^{3} z^{2}}{61603278561}-\frac{19807570229 \sqrt{2} \rho^{2} z}{584130307695}\right) \\
& +\left(383757256080000 a_{2}-\rho^{2}(1353367360104125 \sqrt{2} \rho z+257897855245984)\right) \\
& \frac{e^{-\frac{15 \pi}{2 \sqrt{2}}} \rho^{3} z^{2}}{50519616682304880}-\frac{e^{-\frac{5 \pi}{\sqrt{2}}} \rho^{2} z}{18475687048500}((251929337505 \rho z+178892943548 \sqrt{2}) \\
& \left.\rho^{2}-392700 a_{2}(846537 \sqrt{2}-2121875 \rho z)\right)+\frac{8 e^{-\frac{5 \pi}{2 \sqrt{2}}} \rho}{207306039612375} \\
& \left(8415 a_{2}(49013145 \sqrt{2} \rho z+32779864)-848 \rho^{2}(810462369 \sqrt{2} \rho z+238117880)\right) \\
& -\frac{8}{633798675}(1904085 \sqrt{2} \pi-617344) \rho^{3}+\frac{20581329390625 \rho^{7} z^{4}}{886732746729984} \\
& -\frac{1734375 e^{-\frac{35 \pi}{2 \sqrt{2}}} \rho^{7} z^{4}}{72939328}+\frac{775 e^{-\frac{25 \pi}{2 \sqrt{2}}} \rho^{6} z^{3}(17274375 \rho z+10098404 \sqrt{2})}{374185614528} \\
& +\frac{25 e^{-\frac{15 \pi}{\sqrt{2}}} \rho^{6} z^{3}(376440625 \rho z-293279662 \sqrt{2})}{2259931934304}+\frac{1929033295603982130425 \rho^{6} z^{3}}{53312229133225758319536 \sqrt{2}} \\
& +\frac{12415915880034901 \rho^{5} z^{2}}{926192972508922800}+\frac{114200436283 \sqrt{2} \rho^{4} z}{3154303661553}+\frac{e^{-5 \sqrt{2} \pi} \rho^{5} z^{2}}{297952783603200} \\
& (1589668853472-625 \rho z(18770709375 \rho z+4278131824 \sqrt{2})) \text {, } \\
& I_{2}(\mathbf{z})=e^{-\frac{5 \pi}{2 \sqrt{2}}}\left(\frac{40832 a_{2}^{2} z}{1049427 \rho^{2}}+a_{2}\left(-\frac{49408 \sqrt{2}}{828495 \rho}-\frac{252934685856925 \rho^{2} z^{3}}{2721386433710736}\right.\right.
\end{aligned}
$$




$$
\begin{aligned}
& \left.+\frac{20064998592791 \sqrt{2} \rho z^{2}}{450364467232845}+\left(\frac{9646 \sqrt{2} \pi}{165699}-\frac{925690614976}{7101967672845}\right) z\right)+\frac{2908160 \sqrt{2} \rho}{51863787} \\
& -\frac{5527550890625 \rho^{6} z^{5}}{98525860747776}-\frac{3475436330910009942475 \rho^{5} z^{4}}{47388648118422896284032 \sqrt{2}} \\
& -\frac{115148537639610717947 \rho^{4} z^{3}}{88107811281801317041200}-\frac{196462205901224732 \sqrt{2} \rho^{3} z^{2}}{4154612210222995125}+\left(-\frac{2734 \sqrt{2} \pi}{43605}\right. \\
& \left.\left.+\frac{18720891867514208}{158821303067832735}\right) \rho^{2} z\right)-\frac{4 e^{-\frac{5 \pi}{\sqrt{2}}} z}{3970532576695818375 \rho^{2}}(38622216259836000 \\
& a_{2}^{2}-5350 a_{2} \rho^{2}(13391448585291 \sqrt{2} \rho z+24183667316248) \\
& \left.+\rho^{4}(68542401952527177 \sqrt{2} \rho z+117005574171963800)\right) \\
& +\frac{e^{-\frac{25 \pi}{2 \sqrt{2}}}}{629011432051200} \rho^{2} z^{3}\left(162501300000 a_{2}+\rho^{2}(35625 \rho z(2487684375 \rho z\right. \\
& +1986367162 \sqrt{2})+10845743592224))+\frac{256 \sqrt{2}\left(60409 a_{2}-56800 \rho^{2}\right)}{259318935 \rho}+e^{-\frac{15 \pi}{\sqrt{2}}} \\
& \left(-\frac{\rho^{4} z^{3}(2378225 \rho z(2055650625 \rho z+1197920846 \sqrt{2})+63652108192902)}{25213764854807496}\right. \\
& \left.+\frac{72275 a_{2} \rho^{2} z^{3}}{2990163}\right)+\frac{e^{-5 \sqrt{2} \pi} \rho z^{2}}{25891303549681251000}\left(\rho^{2}(205 \rho z(-1072261547055142\right. \\
& +5052665015015625 \sqrt{2} \rho z)+21417809054549248 \sqrt{2})-1648200 a_{2} \\
& (2177119524375 \rho z+379694102864 \sqrt{2}))+\frac{e^{-\frac{15 \pi}{2 \sqrt{2}}} \rho z^{2}}{3695137409700}(765285440625 \\
& \left.a_{2} \rho z-342014627020 \sqrt{2} a_{2}+426831261472 \sqrt{2} \rho^{2}-18184717070 \rho^{3} z\right) \\
& +\frac{12671875 e^{-10 \sqrt{2} \pi} \rho^{6} z^{5}}{109408992}-\frac{25 e^{-\frac{35 \pi}{2 \sqrt{2}}} \rho^{5} z^{4}(4858486875 \rho z+2058207116 \sqrt{2})}{18079455474432}, \\
& J_{1}(\mathbf{z})=\frac{e^{-\frac{12 \pi}{\sqrt{5}}} z^{2}}{3397781520000 \rho}(\rho(21(2005165908 \sqrt{5}+3889750015) \rho \\
& \left.-2465(3703027 \sqrt{5}+5698606) z)-6211800(193 \sqrt{5}-210) a_{2}\right) \\
& \frac{17722324487957040 \rho^{2}}{5742033134098080960000 \rho}\left(180 \pi \sqrt{5}\left(8 a_{2}-19 \rho^{2}\right)-10(123 \sqrt{5}+520) a_{2}\right. \\
& \left.+(956 \sqrt{5}+5625) \rho^{2}\right)-1212044225 z^{2}\left(962336(4577 \sqrt{5}+1890) a_{2}\right. \\
& \left.-3(10806179752 \sqrt{5}+24675585383) \rho^{2}\right)+325976240 \rho z(3(83295503384 \sqrt{5} \\
& \left.+95313187411) \rho^{2}-10105(13907113 \sqrt{5}+9542762) a_{2}\right)+(7 \sqrt{5}+2) z^{4}
\end{aligned}
$$




$$
\begin{aligned}
& 156788319704681160+8383108(1115434951621 \sqrt{5}+1966086632830) \rho z^{3} \\
& +\frac{e^{-\frac{8 \pi}{\sqrt{5}}} z}{2340848160000 \rho}\left(37600 a_{2}(12(14879 \sqrt{5}+149) \rho+79849 \sqrt{5} z)+3 \rho^{2}\right. \\
& (-47(238651676 \sqrt{5}+506116445) z+240(30405896 \sqrt{5}+66489385) \rho)) \\
& +\frac{e^{-\frac{4 \pi}{\sqrt{5}}}}{6144012000}\left(215 a_{2}(882(123 \sqrt{5}+520) \rho+(146029 \sqrt{5}+155618) z)\right. \\
& \left.-3 \rho^{2}(6321(956 \sqrt{5}+5625) \rho+(48206680 \sqrt{5}+75128363) z)\right) \\
& -\frac{289(7 \sqrt{5}+10) e^{-\frac{28 \pi}{\sqrt{5}}} z^{4}}{10584000 \rho}+\frac{17 e^{-4 \sqrt{5} \pi} z^{3}}{2302020000 \rho}(4930(7 \sqrt{5}+6) z \\
& -3(107371 \sqrt{5}+148485) \rho)-\frac{17(3621 \sqrt{5}+6202) e^{-\frac{24 \pi}{\sqrt{5}}} z^{3}}{168966000}+\frac{e^{-\frac{16 \pi}{\sqrt{5}}} z^{2}}{12519360000 \rho} \\
& \left(-23(2645856 \sqrt{5}+4997225) \rho^{2}-3190560 \sqrt{5} z^{2}+13600(3499 \sqrt{5}+4766) \rho z\right) \text {, } \\
& J_{2}(\mathbf{z})=\frac{e^{-\frac{4 \pi}{\sqrt{5}}}}{312940805808345412320000 \rho^{2}}(533259729244256 z(40250(5 \sqrt{5}+36) \\
& a_{2}^{2}+690(2268 \pi \sqrt{5}-2525 \sqrt{5}+27760) a_{2} \rho^{2}+3(4223690 \sqrt{5}-17388(379 \sqrt{5} \\
& \left.+410) \pi+7544115) \rho^{4}\right)+315385039867317120 \rho^{3}\left(735(35-11 \sqrt{5}) a_{2}+\rho^{2}\right) \\
& (14247 \sqrt{5}-32740)-1509860806 z^{3}\left((295448712792 \sqrt{5}+3357245264653) \rho^{2}\right. \\
& \left.+300730(723208 \sqrt{5}-1530333) a_{2}\right)-15071648 \rho z^{2}\left(a_{2} 464830(14106353 \sqrt{5}\right. \\
& \left.-298387900)+21(16273193039599 \sqrt{5}+23844169989280) \rho^{2}\right)+(1583932 \sqrt{5} \\
& \left.-3899715) 50341483586103 z^{5}+(1249367048959 \sqrt{5}-5521411126760) \rho z^{4}\right) \\
& 234727024-\frac{e^{-\frac{8 \pi}{\sqrt{5}}} z}{353280690000 \rho^{2}}\left(24230500(5 \sqrt{5}+36) a_{2}^{2}+14835 a_{2} \rho(140(5552\right. \\
& -505 \sqrt{5}) \rho+(31424 \sqrt{5}+72755) z)+21 \rho^{3}(430(844738 \sqrt{5}+1508823) \rho \\
& -23(30613883 \sqrt{5}+55472705) z))+\frac{\rho}{992250}\left(735(11 \sqrt{5}-35) a_{2}+(32740\right. \\
& \left.-14247 \sqrt{5}) \rho^{2}\right)+\frac{e^{-\frac{12 \pi}{\sqrt{5}}} z^{2}}{2048242140000 \rho^{2}}\left(235 a_{2}(42(272816 \sqrt{5}+172415) \rho\right. \\
& +5185(2630 \sqrt{5}-6993) z)+6 \rho^{2}(47(26214378 \sqrt{5}+238047761) z-35 \rho \\
& (300370783 \sqrt{5}+736870135)))-\frac{e^{-\frac{16 \pi}{\sqrt{5}}} z^{2}}{5582069640000 \rho^{2}}\left(13340 a_{2}(14(133175\right.
\end{aligned}
$$




$$
\begin{aligned}
& -9586 \sqrt{5}) \rho+765(217 \sqrt{5}-1104) z)+\rho\left(-10440 \rho^{2}(2887157 \sqrt{5}+12737871)\right. \\
& \left.\left.+79373(94964 \sqrt{5}-408025) z^{2}+46(322207806 \sqrt{5}+5283539111) \rho z\right)\right) \\
& +\frac{17 e^{-\frac{24 \pi}{\sqrt{5}} z^{3}}}{6906060000 \rho^{2}}\left(406\left(5(17 \sqrt{5}-72) a_{2}+3(51 \sqrt{5}+602) \rho^{2}\right)+8874(14 \sqrt{5}\right. \\
& \left.-45) z^{2}+1575(1677-274 \sqrt{5}) \rho z\right)+\frac{e^{-4 \sqrt{5} \pi} z^{3}}{4776135840000 \rho^{2}}(5014((303480 \sqrt{5} \\
& \left.+23965123) \rho^{2}-2550(76 \sqrt{5}-105) a_{2}\right)-1102535(2092 \sqrt{5}-4827) z^{2}+476 \\
& (17544628 \sqrt{5}-103898005) \rho z)+\frac{289 e^{-\frac{32 \pi}{\sqrt{5}}}(24-7 \sqrt{5}) z^{5}}{10584000 \rho^{2}}+\frac{17 e^{-\frac{28 \pi}{\sqrt{5}}} z^{4}}{277104240000 \rho^{2}} \\
& (12665(146 \sqrt{5}-205) z-10496(424 \sqrt{5}-3405) \rho) .
\end{aligned}
$$

\section{Acknowledgements}

We thank to the reviewers for their comments and suggestions which help us to improve this paper. The first author is supported by CNPq grant number 248501/2013-5. The second author is partially supported by a FEDERMINECO grant MTM2016-77278-P, a MINECO grant MTM2013-40998-P, and an AGAUR grant number 2014SGR-568.

\section{References}

[1] C. P. Silva, Shil'nikov's theorem-a tutorial, IEEE Transactions on Circuits and Systems I: Fundamental Theory and Applications 40 (10) (1993) 675682.

[2] H. Posh, W. Hoover, F. Vesely, Canonical dynamics of the nosé oscillator: Stability, Order, and Chaos. Phys. Rev. A 33 (1986) 4253.

[3] Z. Wei, Dynamical behaviors of a chaotic system with no equilibria, Physics Letters A 376 (2) (2011) 102-108.

[4] X. Wang, G. Chen, Constructing a chaotic system with any number of equilibria, Nonlinear Dynamics 71 (3) (2013) 429-436.

[5] S. Jafari, J. Sprott, S. M. R. H. Golpayegani, Elementary quadratic chaotic flows with no equilibria, Physics Letters A 377 (9) (2013) 699-702.

[6] V.-T. Pham, S. Vaidyanathan, C. Volos, S. Jafari, Hidden attractors in a chaotic system with an exponential nonlinear term, The European Physical Journal Special Topics 224 (8) (2015) 1507-1517.

[7] S. Vaidyanathan, C. Volos, Analysis and adaptive control of a novel 3-d conservative no-equilibrium chaotic system, Archives of Control Sciences 25 (3) (2015) 333-353. 
[8] Z. Wei, R. Wang, A. Liu, A new finding of the existence of hidden hyperchaotic attractors with no equilibria, Mathematics and Computers in Simulation 100 (2014) 13-23.

[9] J. C. Sprott, Elegant chaos: algebraically simple chaotic flows, World Scientific, 2010.

[10] T. Carvalho, R. D. Euzebio, J. Llibre, D. J. Tonon, Detecting periodic orbits in some $3 \mathrm{~d}$ chaotic quadratic polynomial differential systems, Discrete and continuous dynamical systems series B 21 (2016) 1-11.

[11] J. A. Sanders, F. Verhulst, J. A. Murdock, Averaging methods in nonlinear dynamical systems, Vol. 59, Springer, 2007.

[12] A. Buică, J. Giné, J. Llibre, A second order analysis of the periodic solutions for nonlinear periodic differential systems with a small parameter, Physica D: Nonlinear Phenomena 241 (5) (2012) 528-533.

[13] J. Llibre, D. D. Novaes, Improving the averaging theory for computing periodic solutions of the differential equations, Zeitschrift für angewandte Mathematik und Physik 66 (4) (2015) 1401-1412.

[14] M. R. Cândido, J. Llibre, New results on averaging theory and applications, Zeitschrift für angewandte Mathematik und Physik 67 (4) (2016) 95-106.

[15] M. R. Cândido, J. Llibre, D. D. Novaes, Persistence of periodic solutions for higher order perturbed differential systems via lyapunov-schmidt reduction, Nonlinearity (2017) to appear. 